\title{
Random Dynamical Systems for Stochastic Evolution Equations Driven by Multiplicative Fractional Brownian Noise with Hurst Parameters $H \in(1 / 3,1 / 2]^{*}$
}

\author{
María J. Garrido-Atienza ${ }^{\dagger}$, Kening Lư ${ }^{\ddagger}$, and Björn Schmalfuss ${ }^{\S}$
}

\begin{abstract}
We consider the stochastic evolution equation $d u=A u d t+G(u) d \omega, \quad u(0)=u_{0}$ in a separable Hilbert space $V$. Here $G$ is supposed to be three times Fréchet-differentiable and $\omega$ is a trace class fractional Brownian motion with Hurst parameter $H \in(1 / 3,1 / 2]$. We prove the existence of a unique pathwise global solution, and, since the considered stochastic integral does not produce exceptional sets, we are able to show that the above equation generates a random dynamical system.
\end{abstract}

Key words. stochastic PDEs, Hilbert-valued fractional Brownian motion, pathwise solutions

AMS subject classifications. Primary, 60H15; Secondary, 60H05, 60G22, 26A33, 26A42

DOI. $10.1137 / 15 \mathrm{M} 1030303$

1. Introduction. The main aim of this paper is to prove that stochastic evolution equations (SEEs)

$$
d u=A u d t+G(u) d \omega, \quad u(0)=u_{0},
$$

on a separable Hilbert space $V$ generate random dynamical systems. Our interpretation of the solution will be given in the mild form

$$
u(t)=S(t) u_{0}+\int_{0}^{t} S(t-r) G(u(r)) d \omega(r),
$$

assuming that $A$ is a negative operator that generates a semigroup $S$ and $G$ is sufficiently regular. Our purpose is to study this problem if $\omega$ is Hölder-continuous on compact intervals $[0, T]$. If $\omega$ is a regular trajectory, say, $\omega^{\prime} \in L_{\infty}(0, T, V)$, then a classical formulation of the above problem is given by

$$
u(t)=S(t) u_{0}+\int_{0}^{t} S(t-r) G(u(r)) \omega^{\prime}(r) d r .
$$

If $S$ is an analytic semigroup, under typical assumptions on $G$ there exists a unique solution $u \in C_{\gamma}([0, T] ; V)$ if $u_{0} \in D(-A)$, where $\gamma \in(0,1)$ (see [22, Theorem 4.3.1]); indeed for this

\footnotetext{
${ }^{*}$ Received by the editors July 13, 2015; accepted for publication (in revised form) by $\mathrm{T}$. Wanner January 15, 2016; published electronically March 31, 2016.

http://www.siam.org/journals/siads/15-1/M103030.html

${ }^{\dagger}$ Departamento Ecuaciones Diferenciales y Análisis Numérico, Universidad de Sevilla, 41080-Sevilla, Spain (mgarrido@us.es). This author's work was partially supported by FEDER grant MTM2011-22411.

¥Department of Mathematics Brigham Young University, Provo, UT 84602 (klu@math.byu.edu). This author's work was partially supported by NSF0909400.

$\S$ Institut für Stochastik, Friedrich Schiller Universität Jena, 77043, Jena, Germany (bjoern.schmalfuss@ uni-jena.de). This author's work was partially supported by FEDER grant MTM2011-22411.
}

625

Copyright (C) by SIAM. Unauthorized reproduction of this article is prohibited. 
result to hold it is enough that $u_{0} \in D\left((-A)^{\gamma}\right)$. However, $u \in C_{\gamma, \sim}([0, T] ; V)$ if $u_{0} \in V$. This latter space is a suitable modification of the space of $\gamma$-Hölder-continuous functions and will be introduced in section 2 .

So far, the existence of a unique local mild solution to (2) has been obtained in [11], assuming that $u_{0} \in V$. To be more precise, this solution is derived as the path component of a path-area solution pair $(u, v)$ of a system that considers not only the original equation but also a second equation, the latter one being a natural extension of the area object $(u \otimes$ $\omega)$. This pair $(u, v)$ is related to the noisy path $\omega$ by the so-called Chen property. The method presented in [11] can be considered as a generalization of the one developed by $\mathrm{Hu}$ and Nualart [13] in the finite-dimensional setting, but with some nontrivial differences such as, for example, that in the infinite-dimensional framework it is necessary to construct an area element, denoted by $\left(\omega \otimes_{S} \omega\right)$, depending on $\omega$ as well as the semigroup $S$, that satisfies nice properties. The considered (pathwise) integral in [11] was also previously introduced in [13] for ordinary differential equations with a Hölder-continuous noise term. The reasons we have chosen such a pathwise integral will be detailed below. However, another definition to integrate against the fractional Brownian motion ( $\mathrm{fBm}$ ) would be to consider the rough path theory; see, for instance, [6], [7] and the references therein.

In this paper we want to go one step further and establish the existence of a global solution to (2). In comparison to [11], we here replace the regular initial condition of that paper by a less regular one, namely, $u_{0} \in D\left((-A)^{\gamma}\right)$ for suitable $\gamma>0$, and make a slight modification of the phase spaces, which nevertheless still ensures the additivity of the pathwise integral. Then, under additional regularity conditions on $G$ we prove the existence of a global solution to (2). Let us mention that the estimates of the different integrals defining the path-area formulation of our problem are quadratic, and therefore in a first step we are only able to establish the existence of a local solution $\left(u^{1}, v^{1}\right)$ such that the path component $u^{1}$ is defined on a time interval $\left[T_{0}, T_{1}\right]$. However, since in particular $u^{1}\left(T_{1}\right)$ also belongs to $D\left((-A)^{\gamma}\right)$, we can pick it as a new initial condition, and hence we get a new solution $\left(u^{2}, v^{2}\right)$ such that $u^{2}$ is now defined on a time interval $\left[T_{1}, T_{2}\right]$. Proceeding in a similar way, after a finite number of these steps one finally derives the existence of a global solution on $\left[T_{0}, T\right]$ for a given $T>0$ provided that $u_{0} \in D\left((-A)^{\gamma}\right)$. The way in which we finally can establish the existence of a global solution is based in a concatenation procedure. Furthermore, this method also provides the existence of a global solution when $u_{0} \in V$, since roughly speaking what one has to do is to concatenate the local solution obtained in [11] with the aforementioned global one.

Once the existence of a global solution is established, it is quite natural to study whether that solution generates a random dynamical system, which turns out to be a powerful property to analyze the asymptotic behavior of the solution by using all the machinery of the random dynamical systems theory. The reader is referred to [1] for a complete description of that theory.

The fact that an ordinary Itô equation

$$
d u=G(u) d \omega, u(0)=u_{0} \in \mathbb{R}^{d},
$$

where $\omega$ is a finite-dimensional Brownian motion, generates a random dynamical system is due to the flow property; see Kunita [19]. To obtain this flow property one needs to apply Kolmogorov's theorem about the existence of a continuous random field with finite-dimensional 
parameter range. Scheutzow in [23] derived the existence of a random dynamical system from this flow property.

Results on the existence and uniqueness for Itô SEEs having as a state space an infinitedimensional separable Banach space are established in Da Prato and Zabczyk [5]. The point is that, unfortunately, it is not known how to obtain stochastic flows for these Itô SEEs, and the main reason is because it is not known how to extend Kolmogorov's theorem to an infinite-dimensional parameter range, which would be appropriate for dealing with SEEs. More precisely, solutions of SEEs are defined almost surely where the exceptional sets depend on the initial condition. But it is complicated to generate a random dynamical system if more than countably many exceptional sets may appear. Nevertheless, there are results getting the random dynamical system for SEEs with very special noise terms, SEEs driven by either additive noise or linear multiplicative noise. These special noises make it possible to transform such an Itô SEE into a pathwise evolution equation which is appropriate to generate a random dynamical system. More general than the random dynamical system property is the flow property. For finite-dimensional differential equations with general integrator (for instance, the Brownian motion) the flow property is investigated in Lyons and Qian [20].

The ansatz in this paper is quite different. We shall consider a pathwise integral which is well-defined for any $\beta^{\prime}$-Hölder-continuous integrator $\left(1 / 3<\beta^{\prime}<1 / 2\right)$ if the integrand is sufficiently regular. As we will explain below, this regularity can be described in terms of a modified space of Hölder-continuous functions. Furthermore, by the choice of that integral the unique solution has pathwise character, which means that we can avoid exceptional sets depending on the initial states and making it possible to investigate whether it generates a random dynamical system. We refer to [9] for a short and recent announcement of our results.

The article is organized as follows. In section 2 we collect tools from functional analysis that will be applied later. In particular, we introduce analytic semigroups, special nonlinear operators, tensor products, and function spaces given by modification of Hölder-continuous functions. In section 3 we at first introduce fractional derivatives allowing us to define an integral with a Hölder-continuous integrator, and we give the main properties of such an integral. In section 4 we define the mild path-area solution of a SEE. In particular, we formulate an operator equation whose fixed point presents this kind of (local) solution and mention an approximation result. In section 5 we investigate how the local solution can be extended to any interval $[0, T]$ by means of the concatenation method. For this type of solution we can avoid state-dependent exceptional sets, which is then the key to proving in section 6 the existence of a random dynamical system generated by a SEE. Finally, in section 7, a stochastic partial differential equation is discussed as an example of a SEE generating a mild path-area solution.

2. Preliminaries. In the following we denote by $V, \hat{V}$, or $\tilde{V}$ separable Hilbert spaces. Then as usual $L(V, \hat{V})(L(V))$ denotes the Banach space of linear operators from $V$ to $\hat{V}$ (V to itself) and $L_{2}(V, \hat{V})$ is the separable Hilbert space of Hilbert-Schmidt operators from $V$ to $\hat{V}$.

Let $S$ be an analytic semigroup on $V$ with generator $A$. $-A$ is supposed to be symmetric, positive and have an inverse which is compact. Then $-A$ has a discrete spectrum $0<\lambda_{1} \leq$ $\lambda_{2} \leq \cdots$ of finite multiplicity tending to $+\infty$. The associated eigenelements $\left(e_{i}\right)_{i \in \mathbb{N}}$ are written such that they form a complete orthonormal system of $V$. In addition, we can define 
the associated fractional powers of $-A$ denoted by $(-A)^{\delta}, \delta \in \mathbb{R}$, with norm $|x|_{D\left((-A)^{\delta}\right)}:=$ $\left|(-A)^{\delta} x\right| ;$ see Pazy [22, section 2.6]. We denote $V_{\delta}=D\left((-A)^{\delta}\right)$.

In the next lemma we collect some useful properties of the semigroup $S$.

Lemma 1. If $T>0,0 \leq \delta \leq \gamma, \sigma-\theta \in[0,1]$, then there exists $c>0$ such that for $t \in[0, T]$

$$
\begin{gathered}
\|S(t)\|_{L\left(V_{\delta}, V_{\gamma}\right)}=\left\|(-A)^{\gamma} S(t)\right\|_{L\left(V_{\delta}, V\right)} \leq c t^{\delta-\gamma}, \\
\|S(t)-\mathrm{id}\|_{L\left(V_{\sigma}, V_{\theta}\right)} \leq c t^{\sigma-\theta} .
\end{gathered}
$$

From these two properties, one can easily deduce that for any $\nu, \mu \in[0,1], 0 \leq \delta \leq \gamma+\nu$, $0 \leq \rho$, and $T>0$ there exists a constant $c>0$ such that for $0 \leq q<r<s<t \leq T$ we have that

$$
\begin{aligned}
& \|S(t-r)-S(t-q)\|_{L\left(V_{\delta}, V_{\gamma}\right)} \leq c(r-q)^{\nu}(t-r)^{-\nu-\gamma+\delta} \\
& \|S(t-r)-S(s-r)-S(t-q)+S(s-q)\|_{L\left(V_{\rho}\right)} \\
& \quad \leq c(t-s)^{\mu}(r-q)^{\nu}(s-r)^{-(\nu+\mu)} .
\end{aligned}
$$

Denote by $(V \otimes V,\|\cdot\|)$ the separable Hilbert space of tensor products of $V$; see Kadinson and Ringrose [15]. Moreover, for $x, y \in V$ the element $\left(x \otimes_{V} y\right)$ denotes the rank-one tensor product. In particular, $\left(\left(e_{i} \otimes_{V} e_{j}\right)\right)_{i, j \in \mathbb{N}}$ is a complete orthonormal system for $V \otimes V$ if $\left(e_{i}\right)_{i \in \mathbb{N}}$ is a complete orthonormal system of $V$.

Consider a bilinear operator $B \in L_{2}(V \times V, \hat{V})$ where

$$
\|B\|_{L_{2}(V \times V, \hat{V})}^{2}=\sum_{i, j}\left|B\left(e_{i}, e_{j}\right)\right|_{\hat{V}}^{2}<\infty .
$$

Then it is possible to extend $B$ to a linear continuous operator $\hat{B} \in L_{2}(V \otimes V, \hat{V})$. More precisely, there exists a weak Hilbert-Schmidt mapping $p: V \times V \rightarrow V \otimes V$ such that $p\left(e_{i}, e_{j}\right)=\left(e_{i} \otimes_{V} e_{j}\right)$ and $\hat{B}$ is determined by factorization: $B=\hat{B} p$, and

$$
\|\hat{B}\|_{L_{2}(V \otimes V, \hat{V})}^{2}=\sum_{i, j}\left|\hat{B}\left(e_{i} \otimes_{V} e_{j}\right)\right|_{\hat{V}}^{2}=\sum_{i, j}\left|B\left(e_{i}, e_{j}\right)\right|_{\hat{V}}^{2}=\|B\|_{L_{2}(V \times V, \hat{V})}^{2} .
$$

For these properties we refer to [15]. In the following we will write for $\hat{B}$ the symbol of the original $B$.

Let $\hat{V}$ be a subspace of $V$. Consider now $G$ to be a Frećhet-differentiable mapping

$$
V \ni u \mapsto G(u) \in L_{2}(V, \hat{V})
$$

with derivative

$$
V \ni u \mapsto D G(u) \in L_{2}\left(V, L_{2}(V, \hat{V})\right) \cong L_{2}(V \times V, \hat{V}),
$$

and therefore $D G(u)$ can be interpreted as an element of $L_{2}(V \otimes V, \hat{V})$.

In what follows $|\cdot|$ represents the norm of $V$. 
Let us formulate some assumptions and properties for $G$.

Lemma 2. Assume that the mapping $G: V \rightarrow L_{2}(V, \hat{V})$ is bounded and three times continuously Fréchet-differentiable with bounded first, second, and third derivatives $D G(u), D^{2} G(u)$, and $D^{3} G(u)$, for $u \in V$. Let us denote, respectively, by $c_{G}, c_{D G}, c_{D^{2} G}$, and $c_{D^{3} G}$ the bounds for $G, D G, D^{2} G$, and $D^{3} G$. Then, for $u_{1}, u_{2}, v_{1}, v_{2} \in V$, we have

- $\left\|G\left(u_{1}\right)\right\|_{L_{2}(V, \hat{V})} \leq c_{G}$,

- $\left\|G\left(u_{1}\right)-G\left(v_{1}\right)\right\|_{L_{2}(V, \hat{V})} \leq c_{D G}\left|u_{1}-v_{1}\right|$,

- $\left\|D G\left(u_{1}\right)-D G\left(v_{1}\right)\right\|_{L_{2}(V \times V, \hat{V})} \leq c_{D^{2} G}\left|u_{1}-v_{1}\right|$,

- $\left\|G\left(u_{1}\right)-G\left(u_{2}\right)-D G\left(u_{2}\right)\left(u_{1}-u_{2}\right)\right\|_{L_{2}(V, \hat{V})} \leq c_{D^{2} G}\left|u_{1}-u_{2}\right|^{2}$,

- $\left\|G\left(u_{1}\right)-G\left(v_{1}\right)-\left(G\left(u_{2}\right)-G\left(v_{2}\right)\right)\right\|_{L_{2}(V, \hat{V})} \leq c_{D G}\left|u_{1}-v_{1}-\left(u_{2}-v_{2}\right)\right|$

$+c_{D^{2} G}\left|u_{1}-u_{2}\right|\left(\left|u_{1}-v_{1}\right|+\left|u_{2}-v_{2}\right|\right)$,

- $\left\|D G\left(u_{1}\right)-D G\left(v_{1}\right)-\left(D G\left(u_{2}\right)-D G\left(v_{2}\right)\right)\right\|_{L_{2}(V \times V, \hat{V})}$ $\leq c_{D^{2} G}\left|u_{1}-v_{1}-\left(u_{2}-v_{2}\right)\right|+c_{D^{3} G}\left|u_{1}-u_{2}\right|\left(\left|u_{1}-v_{1}\right|+\left|u_{2}-v_{2}\right|\right)$,

- $\left\|G\left(u_{1}\right)-G\left(u_{2}\right)-D G\left(u_{2}\right)\left(u_{1}-u_{2}\right)-\left(G\left(v_{1}\right)-G\left(v_{2}\right)-D G\left(v_{2}\right)\left(v_{1}-v_{2}\right)\right)\right\|_{L_{2}(V, \hat{V})}$ $\leq c_{D^{2} G}\left(\left|u_{1}-u_{2}\right|+\left|v_{1}-v_{2}\right|\right)\left|u_{1}-v_{1}-\left(u_{2}-v_{2}\right)\right|$ $+c_{D^{3} G}\left|v_{1}-v_{2}\right|\left|u_{2}-v_{2}\right|\left(\left|u_{1}-u_{2}\right|+\left|u_{1}-v_{1}-\left(u_{2}-v_{2}\right)\right|\right)$.

The proof of these properties is standard. We refer partially to [21] and for the last inequality to [13, p. 2716].

Let us introduce some function spaces. For $\beta \in(0,1)$, denote by $C_{\beta}([0, T] ; V)$ the space of $\beta$-Hölder-continuous functions with seminorm

$$
\|u\|_{\beta}=\sup _{0 \leq s<t \leq T} \frac{|u(t)-u(s)|}{(t-s)^{\beta}} .
$$

In this space we can consider the usual norm

$$
\|u\|_{\beta}=\|u\|_{C}+\|u\|_{\beta}, \quad \text { with }\|u\|_{C}=\sup _{0 \leq s \leq T}|u(s)|,
$$

but this norm is equivalent to the norm given by $|u(0)|+\|u\|_{\beta}$. This latter one is the norm with which $C_{\beta}([0, T] ; V)$ is equipped.

A modification of the space of Hölder-continuous functions is given by $C_{\beta, \sim}([0, T] ; V)$ with norm

$$
\|u\|_{\beta, \sim}=\|u\|_{C}+\|u\|_{\beta, \sim} \text { with }\|u\|_{\beta, \sim}=\sup _{0<s<t \leq T} s^{\beta} \frac{|u(t)-u(s)|}{(t-s)^{\beta}} .
$$

Lemma 3. $C_{\beta, \sim}([0, T] ; V)$ is a Banach space.

The proof can be found in Chen et al. [4].

Copyright (C) by SIAM. Unauthorized reproduction of this article is prohibited. 
For the following we suppose that $0<\beta<\beta^{\prime}<1 / 2$. Let $\Delta_{0, T}$ be the triangle $\{(s, t) \in$ $\left.\mathbb{R}^{2}: 0<s \leq t \leq T\right\}$ and $\bar{\Delta}_{0, T}$ its closure in $\mathbb{R}^{2}$. Denote by $C_{\beta+\beta^{\prime}}\left(\bar{\Delta}_{0, T} ; V \otimes V\right)$ the Banach space of continuous functions defined on $\bar{\Delta}_{0, T}$ that are zero for $s=t$ and with norm

$$
\|v\|_{\beta+\beta^{\prime}}=\sup _{0 \leq s<t \leq T} \frac{\|v(s, t)\|}{(t-s)^{\beta+\beta^{\prime}}}<\infty
$$

A modification of this space is given by $C_{\beta+\beta^{\prime}, \sim}\left(\Delta_{0, T} ; V \otimes V\right)$, consisting of continuous functions $v$ defined on $\Delta_{0, T}$ that are zero for $0<s=t$ such that

$$
\|v\|_{\beta+\beta^{\prime}, \sim}=\sup _{0<s<t \leq T} s^{\beta} \frac{\|v(s, t)\|}{(t-s)^{\beta+\beta^{\prime}}}<\infty .
$$

These functions may not be defined for $s=0$ and may have a singularity for $(s, t)$, where $s=0$. Replacing $V \otimes V$ by $\mathbb{R}$ an example for an element of this space is $(s, t) \mapsto s^{-\beta}(t-s)^{\beta+\beta^{\prime}}$.

Lemma 4. The spaces $C_{\beta+\beta^{\prime}}\left(\bar{\Delta}_{0, T} ; V \otimes V\right)$ and $C_{\beta+\beta^{\prime}, \sim}\left(\Delta_{0, T} ; V \otimes V\right)$ are Banach spaces.

The proof of this result is similar to that of Lemma 3 and hence we omit it.

3. Fractional pathwise integrals. In this section we introduce a $V$-valued integral where the integrator is not of bounded variation but Hölder-continuous. This gives us an infinitedimensional version of the Young integral. For this purpose we introduce $V$-valued fractional derivatives.

Let $F, \xi$ be sufficiently regular functions on $[s, t]$. For $\alpha \in(0,1)$ we define the right-handside fractional derivative of order $\alpha$ of $F$ and the left-hand-side fractional derivative of order $1-\alpha$ of $\xi_{t-}(\cdot):=\xi(\cdot)-\xi(t)$, given for $0 \leq s \leq r \leq t$ by the expressions

$$
\begin{aligned}
D_{s+}^{\alpha} F[r] & =\frac{1}{\Gamma(1-\alpha)}\left(\frac{F(r)}{(r-s)^{\alpha}}+\alpha \int_{s}^{r} \frac{F(r)-F(q)}{(r-q)^{1+\alpha}} d q\right), \\
D_{t-}^{1-\alpha} \xi_{t-}[r] & =\frac{(-1)^{1-\alpha}}{\Gamma(\alpha)}\left(\frac{\xi(r)-\xi(t)}{(t-r)^{1-\alpha}}+(1-\alpha) \int_{r}^{t} \frac{\xi(r)-\xi(q)}{(q-r)^{2-\alpha}} d q\right) .
\end{aligned}
$$

Here $\Gamma(\cdot)$ denotes the Gamma function.

The following theorem states regularity conditions on $F, \xi$ such that the Young integral exists.

Theorem 5. Assume $\beta>\alpha$ and $\alpha+\beta^{\prime}>1$. Let $\tilde{V}, \hat{V}$ be two separable Hilbert spaces with complete orthonormal bases $\left(\tilde{e}_{i}\right)_{i \in \mathbb{N}}$ and $\left(\hat{e}_{j}\right)_{j \in \mathbb{N}}$, respectively. Let $F \in C_{\beta, \sim}\left([0, T] ; L_{2}(\tilde{V}, \hat{V})\right)$, $\xi \in C_{\beta^{\prime}}([0, T] ; \tilde{V})$ and assume

$$
r \mapsto\left\|D_{s+}^{\alpha} F[r]\right\|_{L_{2}(\tilde{V}, \hat{V})}\left|D_{t-}^{1-\alpha} \xi_{t-}[r]\right|_{\tilde{V}}
$$

is Lebesgue integrable. Then for $0 \leq s \leq r \leq t \leq T$ the integral

$$
\int_{s}^{t} F(r) d \xi(r):=(-1)^{\alpha} \sum_{j}\left(\sum_{i} \int_{s}^{t} D_{s+}^{\alpha}\left(\hat{e}_{j}, F(\cdot) \tilde{e}_{i}\right)_{\hat{V}}[r] D_{t-}^{1-\alpha}\left(\tilde{e}_{i}, \xi_{t-}(\cdot)\right)_{\tilde{V}}[r] d r\right) \hat{e}_{j}
$$

Copyright (C) by SIAM. Unauthorized reproduction of this article is prohibited. 
is well-posed and satisfies

$$
\left|\int_{s}^{t} F(r) d \xi(r)\right|_{\hat{V}} \leq \int_{s}^{t}\left\|D_{s+}^{\alpha} F[r]\right\|_{L_{2}(\tilde{V}, \hat{V})}\left|D_{t-}^{1-\alpha} \xi_{t-}[r]\right|_{\tilde{V}} d r<\infty .
$$

For the proof we refer to [11]. In particular, we have reduced the definition of this Hilbert space valued integral to an infinite sum of one-dimensional Young integrals.

Corollary 6. Under the assumptions of Theorem 5 , for any $T>0$ there exists $c>0$ such that for any $F \in C_{\beta, \sim}\left([0, T] ; L_{2}(\tilde{V}, \hat{V})\right), \xi \in C_{\beta^{\prime}}([0, T] ; \tilde{V})$, and $0 \leq s<t \leq T$

$$
\left|\int_{s}^{t} F(r) d \xi(r)\right|_{\hat{V}} \leq c(t-s)^{\beta^{\prime}}\|\xi\|_{\beta^{\prime}}\left(\|F\|_{C}+\|F\|_{\beta, \sim}\right)<\infty .
$$

Similarly, if $F \in C_{\beta}\left([0, T] ; L_{2}(\tilde{V}, \hat{V})\right), \xi \in C_{\beta^{\prime}}([0, T] ; \tilde{V})$, then we get

$$
\left|\int_{s}^{t} F(r) d \xi(r)\right|_{\hat{V}} \leq c(t-s)^{\beta^{\prime}}\|\xi\|_{\beta^{\prime}}\left(\|F\|_{C}+(t-s)^{\beta}\|F\|_{\beta}\right) .
$$

Note that the first estimate is an immediate consequence of the fact that if $F \in C_{\beta, \sim}([0, T]$; $\left.L_{2}(\tilde{V}, \hat{V})\right)$ with $\alpha+\beta>1$, and $\xi \in C_{\beta^{\prime}}([0, T] ; \tilde{V})$, then

$$
\begin{aligned}
\left\|D_{s+}^{\alpha} F[r]\right\|_{L_{2}(\tilde{V}, \hat{V})} & \leq c \frac{\|F\|_{C}+\|F\|_{\beta, \sim}}{(r-s)^{\alpha}} \\
\left|D_{t-}^{1-\alpha} \xi_{t-}[r]\right|_{\tilde{V}} & \leq c\|\xi\|_{\beta^{\prime}}(t-r)^{\beta^{\prime}+\alpha-1} .
\end{aligned}
$$

The second inequality can be deduced in a similar way.

Suppose now that $\beta \leq \alpha$; then the integral of Theorem 5 does not make sense. To overcome this problem we introduce a modification of the fractional derivatives. More precisely, we introduce a new fractional derivative for a special function $F$ given by the integrand of our original problem (2), so that we set $F(t)=G(u(t))$, where the operator $G$ satisfies the assumptions described in Lemma 2. For that function we consider the compensated fractional derivative defined as

$$
\begin{aligned}
\hat{D}_{s+}^{\alpha} G(u(\cdot))[r]= & \frac{1}{\Gamma(1-\alpha)}\left(\frac{G(u(r))}{(r-s)^{\alpha}}\right. \\
& \left.+\alpha \int_{s}^{r} \frac{G(u(r))-G(u(q))-D G(u(q))(u(r)-u(q))}{(r-q)^{1+\alpha}} d q\right) .
\end{aligned}
$$

For tensor valued elements $v: \Delta_{0, T} \rightarrow V \otimes V$ we define

$$
\mathcal{D}_{t-}^{1-\alpha} v[r]=\frac{(-1)^{1-\alpha}}{\Gamma(\alpha)}\left(\frac{v(r, t)}{(t-r)^{1-\alpha}}+(1-\alpha) \int_{r}^{t} \frac{v(r, q)}{(q-r)^{2-\alpha}} d q\right) .
$$

Below we clarify under which conditions these fractional derivatives are well-defined. We set $\xi=\omega \in C_{\beta^{\prime}}([0, T] ; V)$ satisfying hypothesis (H3) of section 4. If the triplet $u, \omega, v$ satisfies the so-called Chen equality, that is, for $0<s \leq r \leq t$

$$
v(s, r)+v(r, t)+(u(r)-u(s)) \otimes_{V}(\omega(t)-\omega(r))=v(s, t),
$$


then the definition of the pathwise stochastic integral for the current work is the following:

$$
\begin{aligned}
\int_{s}^{t} G(u) d \omega:= & (-1)^{\alpha} \int_{s}^{t} \hat{D}_{s+}^{\alpha} G(u(\cdot))[r] D_{t-}^{1-\alpha} \omega_{t-}[r] d r \\
& -(-1)^{2 \alpha-1} \int_{s}^{t} D_{s+}^{2 \alpha-1} D G(u(\cdot))[r] D_{t-}^{1-\alpha} \mathcal{D}_{t-}^{1-\alpha} v(\cdot, t)[r] d r .
\end{aligned}
$$

Note that this definition is an infinite-dimensional generalization of the concept of integral given in [13].

According to the previous definition, for the integrand of the integral of the left-hand-side we should rather write the term $G_{v}(u)$ instead of $G(u)$, but for the sake of simplification we keep $G(u)$. Furthermore, for sufficiently regular $\omega$ this integral can be rewritten in the sense of Theorem 5 with $F(t)=G(u(t))$ if we replace $v(s, t)$ by

$$
(u \otimes \omega)(s, t)=\int_{s}^{t}(u(r)-u(s)) \otimes_{V} d \omega(r)
$$

see also the beginning of the next section.

Throughout the whole paper, we will make use of the following property: for every $s<t$, $\mu, \nu>-1$,

$$
\int_{s}^{t}(r-s)^{\mu}(t-r)^{\nu} d r=c(t-s)^{\mu+\nu+1}
$$

where $c=B(\mu+1, \nu+1)$ (here $B(\cdot, \cdot)$ denotes the Beta function). The proof is a simple exercise since it follows by making a change of variable and take into account the definition of the Beta function.

For the existence of (9) we have the following result.

Theorem 7. Consider $1 / 3<\beta<\beta^{\prime}<1 / 2$, choose $\beta<\alpha<2 \beta, \alpha+\beta^{\prime}>1, \beta+1>2 \alpha$, and assume that $G$ satisfies the assumptions of Lemma 2. Then for $T>0$ there exists $c>0$ such that for $u \in C_{\beta, \sim}([0, T] ; V), v \in C_{\beta+\beta^{\prime}, \sim}\left(\Delta_{0, T} ; V \otimes V\right)$, and $\omega \in C_{\beta^{\prime}}([0, T] ; V)$ such that (8) is fulfilled, for $0 \leq s \leq t \leq T$

$$
\left|\int_{s}^{t} G(u) d \omega\right|_{\hat{V}} \leq c t^{\beta^{\prime}-\beta}\left(\left(1+\|u\|_{\beta, \sim}^{2}\right)\|\omega\|_{\beta^{\prime}}+\left(1+\|u\|_{\beta, \sim}\right)\|v\|_{\beta+\beta^{\prime}, \sim}\right)(t-s)^{\beta} .
$$

Similarly, with the same choice of the parameters, if $\omega \in C_{\beta^{\prime}}([0, T] ; V), u \in C_{\beta}([0, T] ; V)$, and $v \in C_{\beta+\beta^{\prime}}\left(\Delta_{0, T} ; V \otimes V\right)$ are coupled by (8), then

$$
\left|\int_{s}^{t} G(u) d \omega\right|_{\hat{V}} \leq c t^{\beta^{\prime}-\beta}\left(\left(1+t^{2 \beta}\|u\|_{\beta}^{2}\right)\|\omega\|_{\beta^{\prime}}+t^{\beta}\left(1+t^{\beta}\|u\|_{\beta}\right)\|v\|_{\beta+\beta^{\prime}}\right)(t-s)^{\beta} .
$$

Note that if $u \in C_{\beta, \sim}([0, T] ; V)$, then

$$
\left\|\hat{D}_{s+}^{\alpha} G(u)[r]\right\|_{L_{2}(V, \hat{V})} \leq c \frac{c_{G}+c_{D^{2} G}\|u\|_{\beta, \sim}^{2}}{(r-s)^{\alpha}},
$$

Copyright $\odot$ by SIAM. Unauthorized reproduction of this article is prohibited. 
and if $u \in C_{\beta}([0, T] ; V)$ we get

$$
\left\|\hat{D}_{s+}^{\alpha} G(u)[r]\right\|_{L_{2}(V, \hat{V})} \leq c \frac{c_{G}+c_{D^{2} G}(r-s)^{2 \beta}\|u\|_{\beta}^{2}}{(r-s)^{\alpha}},
$$

hence the estimates of $\left\|\hat{D}_{s+}^{\alpha} G(u)[r]\right\|_{L_{2}(V, \hat{V})}$, together with the estimate (7) for $\xi=\omega$ and the property (11), give the first terms on the left-hand-side of the inequalities of the theorem.

For the second integral we can use Theorem 5 directly replacing $\alpha$ by $2 \alpha-1, G(u(r))$ by $D G(u(r))$ defined on $V \otimes V$, and $\xi(r)$ by $\mathcal{D}_{t-}^{1-\alpha} v(\cdot, t)[r]$. In particular, taking the Chen equality and the regularity into account, in the first case we have

$$
\begin{aligned}
& \left\|D_{s+}^{2 \alpha-1} D G(u)[r]\right\|_{L_{2}(V \otimes V, \hat{V})} \leq c \frac{c_{D G}+c_{D^{2} G}\|u\|_{\beta, \sim}}{(r-s)^{2 \alpha-1}}, \\
& \left\|D_{t-}^{1-\alpha} \mathcal{D}_{t-}^{1-\alpha} v[r]\right\| \leq c\left(\|v\|_{\beta+\beta^{\prime}, \sim}+\|u\|_{\beta, \sim}\|\omega\|_{\beta^{\prime}}\right)(r-s)^{-\beta}(t-r)^{\beta+\beta^{\prime}+2 \alpha-2},
\end{aligned}
$$

and in second case

$$
\begin{aligned}
& \left\|D_{s+}^{2 \alpha-1} D G(u)[r]\right\|_{L_{2}(V \otimes V, \hat{V})} \leq c \frac{c_{D G}+c_{D^{2} G}(r-s)^{\beta}\|u\|_{\beta}}{(r-s)^{2 \alpha-1}} \\
& \left\|D_{t-}^{1-\alpha} \mathcal{D}_{t-}^{1-\alpha} v[r]\right\| \leq c\left(\|v\|_{\beta+\beta^{\prime}}+\|u\|_{\beta}\|\omega\|_{\beta^{\prime}}\right)(t-r)^{\beta+\beta^{\prime}+2 \alpha-2} .
\end{aligned}
$$

More details concerning the proof of this theorem can be found in [11].

To finish this section, we want to emphasize that in contrast to the classical Itô integral which is defined as a limit in probability of Darboux sums with respect to the increments of a Brownian motion, with the above definition there are no exceptional sets of probability which in general depend on the integrand. In this sense our integral is pathwise, allowing us to investigate whether the solution to (1) generates a random dynamical system; see section 6 .

4. Pathwise local solutions of SEEs. In this section we formulate a result about the local existence of solutions to (1) for a noise-path $\omega$ which is in $C_{\beta^{\prime}}([0, T] ; V), \beta^{\prime} \in(1 / 3,1 / 2)$. In particular we formulate conditions such that (2) has a local mild solution when the pathwise (stochastic) integral is defined in the sense of (9). For the proofs of the results given in this section we refer to Garrido-Atienza, Lu, and Schmalfuss [11]. These results generalize those of $\mathrm{Hu}$ and Nualart [13] to the case of a state space which is now an infinite-dimensional Hilbert space.

If $\omega$ were (for instance) a trace class Brownian-motion, according to the theory of Da Prato and Zabczyk [5], (2) would have a unique mild solution provided that $G$ and $S$ satisfy weaker conditions than the ones required in the last section. The corresponding integrals for these equations would be defined in the Itô sense, and this means that they would not be pathwise, which would be an obstacle to generating a random dynamical system. In Maslowski and Nualart [21] these types of equations are solved by fractional techniques for $\beta^{\prime}$-Hölder-continuous integrators with $\beta^{\prime}>1 / 2$, where the integrals have been defined similarly to Theorem 5 , allowing us to introduce a random dynamical system, whose existence has been investigated recently by Chen et al. [4]. However, the integral as introduced in Theorem 5 is not welldefined when the Hölder exponent of the integrator is less than or equal to $1 / 2$, which is the case that we aim to consider in this paper.

Copyright (c) by SIAM. Unauthorized reproduction of this article is prohibited. 
We interpret the solution to (2) as the first component of an element $U=(u, v)$ that satisfies the operation equation

$$
\begin{aligned}
& u=\mathcal{T}_{1}(U), \\
& \mathcal{T}_{1}\left(U, \omega, u_{0}\right)(t)=S(t) u_{0}+(-1)^{\alpha} \int_{0}^{t} \hat{D}_{0+}^{\alpha}(S(t-\cdot) G(u(\cdot)))[r] D_{t-}^{1-\alpha} \omega[r] d r \\
& -(-1)^{2 \alpha-1} \int_{0}^{t} D_{0+}^{2 \alpha-1}(S(t-\cdot) D G(u(\cdot)))[r] D_{t-}^{1-\alpha} \mathcal{D}_{t-}^{1-\alpha} v[r] d r .
\end{aligned}
$$

Note that to define $\mathcal{T}_{1}$ we have applied the definition given in (9), which makes it necessary to set up and solve an equation for the second component $v$ of $U$. In order to do that, we assume for a while that the driving path $\omega$ is smooth enough, giving us the opportunity to know what is the suitable equation to be satisfied for the $v$ component, to later make adequate assumptions to translate the situation to the case of having a Hölder-continuous driving path $\omega$ with Hölder exponent $\beta^{\prime} \in(1 / 3,1 / 2)$. Therefore, suppose that $\omega$ is smooth, so that $v$ is given by (10). For such a smooth $\omega$, combining (2) with (10) we have

$$
\begin{aligned}
(u \otimes \omega)(s, t)= & \int_{s}^{t}(S(\xi-s)-\mathrm{id}) u(s) \otimes_{V} \omega^{\prime}(\xi) d \xi \\
& +\int_{s}^{t} \int_{s}^{\xi} S(\xi-r) G(u(r)) \omega^{\prime}(r) d r \otimes_{V} \omega^{\prime}(\xi) d \xi .
\end{aligned}
$$

Representing the appearing integrals in a fractional sense we obtain

$$
\begin{aligned}
(u \otimes \omega) & (s, t)=(-1)^{\alpha} \int_{s}^{t} D_{s+}^{\alpha}((S(\cdot-s)-\mathrm{id}) u(s))[\xi] \otimes_{V} D_{t-}^{1-\alpha} \omega[\xi] d \xi \\
& -(-1)^{\alpha} \int_{s}^{t} \hat{D}_{s+}^{\alpha} G(u(\cdot))[r] D_{t-}^{1-\alpha}\left(\omega \otimes_{S} \omega\right)(\cdot, t)_{t-}[r] d r \\
& +(-1)^{2 \alpha-1} \int_{s}^{t} D_{s+}^{2 \alpha-1} D G(u(\cdot))[r] D_{t-}^{1-\alpha} \mathcal{D}_{t-}^{1-\alpha}\left(u \otimes\left(\omega \otimes_{S} \omega\right)(t)\right)[r] d r
\end{aligned}
$$

Note that the first integral of (12) has been expressed in (13) in terms of standard fractional derivatives due to the regularity of its integrand; see [11, Lemma 22].

For such smooth $\omega$, the operator $\left(\omega \otimes_{S} \omega\right)$ is given by

$$
\begin{aligned}
L_{2}(V, \hat{V}) \ni E \mapsto E\left(\omega \otimes_{S} \omega\right)(s, t) & =\int_{s}^{t} \int_{s}^{\xi} S(\xi-r) E \omega^{\prime}(r) d r \otimes_{V} \omega^{\prime}(\xi) d \xi \\
& =\int_{s}^{t} \int_{r}^{t} S(\xi-r) E \omega^{\prime}(r) \otimes_{V} \omega^{\prime}(\xi) d \xi d r
\end{aligned}
$$

In particular, $\left(\omega \otimes_{S} \omega\right)$ satisfies the following Chen equality:

$$
\begin{aligned}
& E\left(\omega \otimes_{S} \omega\right)(s, r)+E\left(\omega \otimes_{S} \omega\right)(r, t)+(-1)^{\alpha} \omega_{S}(r, t) S_{\omega}(s, r) E \\
& \quad=E\left(\omega \otimes_{S} \omega\right)(s, t),
\end{aligned}
$$

Copyright (C) by SIAM. Unauthorized reproduction of this article is prohibited. 
where

$$
\begin{aligned}
& e \in V \mapsto \omega_{S}(s, t) e=(-1)^{-\alpha} \int_{s}^{t} S(\xi-s) e \otimes_{V} \omega^{\prime}(\xi) d \xi \\
& E \in L_{2}(V, \hat{V}) \mapsto S_{\omega}(s, t) E=\int_{s}^{t} S(t-r) E \omega^{\prime}(r) d r .
\end{aligned}
$$

The different expressions in (14) and (15) are well-defined when $\omega$ is regular; see [11] for details.

Now let us come back to the original situation in which $\omega \in C_{\beta^{\prime}}([0, T] ; V)$ for $\beta^{\prime} \in$ $(1 / 3,1 / 2)$. Denote by $\omega_{n}$ a piecewise linear (continuous) approximation of $\omega$ with respect to an equidistant partition of length $2^{-n} T$ such that $\omega_{n}(t)=\omega(t)$ for the partition points $t$. For these $\omega_{n}$ the operator $\left(\omega_{n} \otimes_{S} \omega_{n}\right)$ can be defined according to (14). However, when $\omega$ is not smooth we have to give to $\left(\omega \otimes_{S} \omega\right)$ some meaning. This will be made precise in (H3) below.

We assume the following hypothesis $(\mathrm{H})$, which consists of the following three assumptions:

(H1) Let $H \in(1 / 3,1 / 2]$ and let $1 / 3<\beta<\beta^{\prime}<H$. Suppose that there is an $\alpha$ such that $1-\beta<\alpha<2 \beta, \alpha<\frac{\beta+1}{2}$.

(H2) Let $A$ be the generator of an analytic semigroup $S$ and let $G: V \rightarrow L_{2}(V, \hat{V})$ be a nonlinear mapping satisfying the assumptions of Lemma 2, with $\hat{V} \subset V$ such that the embedding operator is Hilbert-Schmidt.

(H3) Let $\left(\omega_{n}\right)_{n \in \mathbb{N}}$ be a sequence of piecewise linear approximations of $\omega$ with respect to an equidistant dyadic $2^{-n}$ partition, with values in $V$ and such that $\left(\left(\omega_{n} \otimes_{S} \omega_{n}\right)\right)_{n \in \mathbb{N}}$ is defined by (14). Assume then that for any $\beta^{\prime}<H$

$$
\lim _{n \rightarrow \infty}\left(\omega_{n},\left(\omega_{n} \otimes_{S} \omega_{n}\right)\right)=\left(\omega,\left(\omega \otimes_{S} \omega\right)\right) \text { in } C_{\beta^{\prime}}([0, T] ; V) \times C_{2 \beta^{\prime}}\left(\bar{\Delta}_{0, T} ; L_{2}\left(L_{2}(V, \hat{V}), V \otimes V\right)\right) .
$$

On account of $(\mathrm{H} 3)$, the object $\left(\omega \otimes_{S} \omega\right)$ is defined as the limit of the sequence $\left(\left(\omega_{n} \otimes_{S}\right.\right.$ $\left.\left.\omega_{n}\right)\right)_{n \in \mathbb{N}}$ in $C_{2 \beta^{\prime}}\left(\bar{\Delta}_{0, T} ; L_{2}\left(L_{2}(V, \hat{V}), V \otimes V\right)\right)$. In the recent paper [10] the authors have proposed two different settings where assumption (H3) is satisfied: the first one considers as driving noise a trace-class $\mathrm{fBm} B^{H}$ with $H \in(1 / 3,1 / 2]$ and values in a Hilbert space, whereas by another less restrictive method, the second one considers an infinite-dimensional trace-class Brownian motion $B^{1 / 2}$. The parameter $H$ in the above assumption (H1) will represent the Hurst parameter of an $\mathrm{fBm}$ in section 6 .

Now we should realize that the structure of the last two integrals of (13) is quite similar to the structure of the two integrals of $\mathcal{T}_{1}$ when replacing $\omega$ by $\left(\omega \otimes_{S} \omega\right)$ and $v$ by $\left(u \otimes\left(\omega \otimes_{S} \omega\right)\right)$. Furthermore, if we write $w(t)=\left(u \otimes\left(\omega \otimes_{S} \omega\right)(t)\right)$, then $w$ can be interpreted by fractional integrals as

$$
\begin{aligned}
\tilde{E} w(t, s, q)= & -\int_{s}^{q} \hat{D}_{s+}^{\alpha} \omega_{S}(\cdot, t) \tilde{E}(u(\cdot)-u(s), \cdot)[r] D_{q-}^{1-\alpha} \omega_{q-}[r] d r \\
& +(-1)^{\alpha-1} \int_{s}^{q} D_{s+}^{2 \alpha-1} \tilde{E}(u(\cdot)-u(s), \cdot)[r] D_{q-}^{1-\alpha} \mathcal{D}_{q-}^{1-\alpha}\left(\omega_{S}(t) \otimes \omega\right)[r] d r \\
& +(-1)^{\alpha-1} \int_{s}^{q} D_{s+}^{2 \alpha-1} \omega_{S}(\cdot, t)[r] \tilde{E} D_{q-}^{1-\alpha} \mathcal{D}_{q-}^{1-\alpha} v(\cdot, q)[r] d r,
\end{aligned}
$$

Copyright (C) by SIAM. Unauthorized reproduction of this article is prohibited. 
where $\tilde{E} \in L_{2}(V \otimes V, \hat{V})$. In addition $w$ satisfies a special form of the Chen equality; see [11]. The previous considerations make it possible to formulate an equation for the second component $v$ of $U$ given by

$$
v=\mathcal{T}_{2}(U)
$$

where the operator $\mathcal{T}_{2}(U)$ is defined by (13) substituting $\left(u \otimes\left(\omega \otimes_{S} \omega\right)\right)$ by $w$, and $w$ satisfies (16).

In what follows, we denote $\mathcal{T}(U)=\left(\mathcal{T}_{1}(U), \mathcal{T}_{2}(U)\right)$. Sometimes we will write

$$
\mathcal{T}\left(U, \omega,\left(\omega \otimes_{S} \omega\right), u_{0}\right)=\left(\mathcal{T}_{1}\left(U, \omega, u_{0}\right), \mathcal{T}_{2}\left(U, \omega,\left(\omega \otimes_{S} \omega\right), u_{0}\right)\right)
$$

to show the different ingredients in the operator. Furthermore, when this operator is defined on $[s, T]$, for $s>0$, instead of on $[0, T]$, later we will denote it by $\mathcal{T}^{s}$. However, to simplify the presentation, when the starting point of the interval is zero we drop the superindex and simply write $\mathcal{T}$.

Now we introduce the phase space where we look for solutions to (1). Take a fixed $\omega \in C_{\beta^{\prime}}([0, T] ; V)$ and consider $\gamma$ such that $\alpha<\gamma<1$. Denote by $\left(W_{0, T},\|\cdot\|_{W}\right)$ the subspace of elements $U=(u, v)$ of the Banach space $C_{\beta, \sim}([0, T] ; V) \times C_{\beta+\beta^{\prime}, \sim}\left(\Delta_{0, T} ; V \otimes V\right)$ such that the Chen equality (8) holds. Let us also consider a subset $\hat{W}_{0, T}$ of this space given by the limit points in this space of the set

$$
\begin{gathered}
\left\{\left(u_{n},\left(u_{n} \otimes \omega_{n}\right)\right): n \in \mathbb{N}, u_{n} \in C_{\gamma}([0, T] ; V), u_{n}(0) \in V_{\gamma}\right. \\
\left.\left(\omega_{n},\left(\omega_{n} \otimes_{S} \omega_{n}\right)\right) \text { satisfies }(\mathrm{H} 3)\right\} .
\end{gathered}
$$

Note that this set of limit points is a subspace of $C_{\beta, \sim}([0, T] ; V) \times C_{\beta+\beta^{\prime}, \sim}\left(\Delta_{0, T} ; V \otimes V\right)$ which is closed. Hence $\hat{W}_{0, T}$ itself is a complete metric space depending on $\omega$ with a metric generated by the norm of $C_{\beta, \sim}([0, T] ; V) \times C_{\beta+\beta^{\prime}, \sim}\left(\Delta_{0, T} ; V \otimes V\right)$ :

$$
d_{\hat{W}}(U, \hat{U})=\|U-\hat{U}\|_{W}=\|u-\hat{u}\|_{\beta, \sim}+\|v-\hat{v}\|_{\beta+\beta^{\prime}, \sim}
$$

for $U, \hat{U} \in \hat{W}_{0, T}$. In addition, elements $U \in \hat{W}_{0, T}$ satisfy the Chen equality (8) with respect to $\omega$.

Remark 8. We would like to emphasize that the choice of the space $\hat{W}_{0, T}$ ensures the following additivity formula:

$$
S(t-\tau) \int_{0}^{\tau} S(\tau-r) G(u(r)) d \omega(r)+\int_{\tau}^{t} S(t-r) G(u(r)) d \omega(r)=\int_{0}^{t} S(t-r) G(u(r)) d \omega(r),
$$

which is derived as a result of the fact that the approximative integrals related to the space $\hat{W}_{0, T}$ enjoy this additivity property. As pointed out in [11], for the original integral it is quite involved to show the additivity, which is necessary to prove the uniqueness of the local solution as well as the Chen equality for the solution of (2).

Definition 9. We say that a solution $U \in \hat{W}_{0, T}$ of the operator equation $U=\mathcal{T}\left(U, \omega,\left(\omega \otimes_{S}\right.\right.$ $\left.\omega), u_{0}\right)$ is a mild path-area solution to (1).

Let us mention some properties of $\mathcal{T}$; see [11] for the proof.

Lemma 10. Suppose (H) holds. 
(i) For two elements $\tilde{U}=(\tilde{u}, \tilde{v}), \hat{U}=(\hat{u}, \hat{v}) \in \hat{W}_{0, T}$ such that $\tilde{u}(0)=\tilde{u}_{0} \in V, \hat{u}(0)=$ $\hat{u}_{0} \in V$,

$$
\begin{aligned}
& \left\|\mathcal{T}\left(\tilde{U}, \omega,\left(\omega \otimes_{S} \omega\right), \tilde{u}_{0}\right)-\mathcal{T}\left(\hat{U}, \omega,\left(\omega \otimes_{S} \omega\right), \hat{u}_{0}\right)\right\|_{W} \\
& \leq \tilde{c}\left|\tilde{u}_{0}-\hat{u}_{0}\right|+c T^{\beta^{\prime}}\left(1+\|\tilde{U}\|_{W}^{2}+\|\hat{U}\|_{W}^{2}\right)\|\tilde{U}-\hat{U}\|_{W},
\end{aligned}
$$

where the constant $c$ depends on $T,\|\omega\|_{\beta^{\prime \prime}},\|\omega\|_{\beta^{\prime}}$ and $\left\|\left(\omega \otimes_{S} \omega\right)\right\|_{2 \beta^{\prime}}$ for $\beta^{\prime}<\beta^{\prime \prime}<H$, and $\tilde{c}$ depends on $\|\omega\|_{\beta^{\prime}}$.

(ii) For $U \in \hat{W}_{0, T}$ we get

$$
\lim _{n \rightarrow \infty}\left\|\mathcal{T}\left(U, \omega_{n},\left(\omega_{n} \otimes_{S} \omega_{n}\right), u_{0}\right)-\mathcal{T}\left(U, \omega,\left(\omega \otimes_{S} \omega\right), u_{0}\right)\right\|_{W}=0 .
$$

This convergence is uniform for $U \in \hat{W}_{0, T}$ contained in a bounded set. In addition, $\mathcal{T}\left(U, \omega,\left(\omega \otimes_{S}\right.\right.$ $\left.\omega), u_{0}\right) \in \hat{W}_{0, T}$.

Next we establish the existence and uniqueness of a local mild solution as well as its regularity.

Theorem 11. Suppose $(\mathrm{H})$ holds. Then for any $u_{0} \in V$ and $\omega \in C_{\beta^{\prime}}([0, T] ; V)$ there exists a time $T_{0}>0$ such that

$$
U=\mathcal{T}(U)
$$

has a unique mild path-area solution $U^{0}=\left(u^{0}, v^{0}\right) \in \hat{W}_{0, T_{0}}$.

Let $\hat{V}=V_{\gamma}$; then for $0<t \leq T_{0}$ we have that $u^{0}(t) \in V_{\gamma}$. In particular,

$$
\left|u^{0}\left(T_{0}\right)\right|_{V_{\gamma}} \leq c \frac{\left|u_{0}\right|}{T_{0}^{\gamma}}+c\left(1+\|\omega\|_{\beta^{\prime}}\right) T_{0}^{\beta^{\prime}}\left(1+\left\|U^{0}\right\|_{W}^{2}\right) .
$$

In addition, there exists a sequence $\left(u_{n}^{0}\right)_{n \in \mathbb{N}}$ with $\lim _{n \rightarrow \infty}\left|u_{n}^{0}(0)-u_{0}\right|=0$ such that

$$
\lim _{n \rightarrow \infty}\left|u^{0}\left(T_{0}\right)-u_{n}^{0}\left(T_{0}\right)\right|_{V_{\gamma}}=0 .
$$

Proof. The first and third parts of the statement have been proven in [11] and hence here we only prove the second one.

Notice that for $\gamma \geq 0$ and any $t>0$ we have

$$
\left|S(t) u_{0}\right|_{V_{\gamma}} \leq c \frac{\left|u_{0}\right|}{t^{\gamma}}
$$

Now, we estimate the $V_{\gamma}$-norm of the integral term:

$$
\begin{aligned}
\int_{0}^{t} S(t-r) G\left(u^{0}(r)\right) d \omega= & (-1)^{\alpha} \int_{0}^{t} \hat{D}_{0+}^{\alpha}\left(S(t-\cdot) G\left(u^{0}(\cdot)\right)\right)[r] D_{t-}^{1-\alpha} \omega_{t-}[r] d r \\
& -(-1)^{2 \alpha-1} \int_{0}^{t} D_{0+}^{2 \alpha-1}\left(S(t-\cdot) D G\left(u^{0}(\cdot)\right)\right)[r] D_{t-}^{1-\alpha} \mathcal{D}_{t-}^{1-\alpha} v^{0}[r] d r \\
= & : I_{1}(t)+I_{2}(t) .
\end{aligned}
$$

Copyright (C) by SIAM. Unauthorized reproduction of this article is prohibited. 
Consider $\alpha^{\prime}>0$ such that $\beta^{\prime}+\alpha>\alpha^{\prime}>\alpha$; then thanks to Lemma 2

$$
\begin{aligned}
\| \hat{D}_{0+}^{\alpha}( & \left.S(t-\cdot) G\left(u^{0}(\cdot)\right)\right)[r] \|_{L_{2}\left(V, V_{\gamma}\right)} \\
\leq & c\left(\frac{\left\|S(t-r) G\left(u^{0}(r)\right)\right\|_{L_{2}\left(V, V_{\gamma}\right)}}{r^{\alpha}}+\int_{0}^{r} \frac{\left\|(S(t-r)-S(t-q)) G\left(u^{0}(r)\right)\right\|_{L_{2}\left(V, V_{\gamma}\right)}}{(r-q)^{\alpha+1}} d q\right. \\
& \left.\quad+\int_{0}^{r} \frac{\left\|S(t-q)\left(G\left(u^{0}(r)\right)-G\left(u^{0}(q)\right)-D G\left(u^{0}(q)\right)\left(u^{0}(r)-u^{0}(q)\right)\right)\right\|_{L_{2}\left(V, V_{\gamma}\right)}}{(r-q)^{\alpha+1}} d q\right) \\
\leq & c\left(\frac{\left\|G\left(u^{0}(r)\right)\right\|_{L_{2}\left(V, V_{\gamma}\right)}}{r^{\alpha}}+\int_{0}^{r} \frac{(r-q)^{\alpha^{\prime}}\left\|G\left(u^{0}(r)\right)\right\|_{L_{2}\left(V, V_{\gamma}\right)}}{(t-r)^{\alpha^{\prime}}(r-q)^{\alpha+1}} d q\right. \\
& \left.\quad+\int_{0}^{r} \frac{\left\|\left(G\left(u^{0}(r)\right)-G\left(u^{0}(q)\right)-D G\left(u^{0}(q)\right)\left(u^{0}(r)-u^{0}(q)\right)\right)\right\|_{L_{2}\left(V, V_{\gamma}\right)}}{(r-q)^{\alpha+1}} d q\right) \\
\leq & c\left(\frac{c_{G}}{r^{\alpha}}+\frac{c_{G}}{(t-r)^{\alpha^{\prime}}} \int_{0}^{r} \frac{(r-q)^{\alpha^{\prime}}}{(r-q)^{\alpha+1}} d q+c_{D^{2} G}\left\|u^{0}\right\|_{\beta, \sim}^{2} \int_{0}^{r} \frac{(r-q)^{2 \beta} q^{-2 \beta}}{(r-q)^{\alpha+1}} d q\right) \\
\leq & c\left(1+\left\|u^{0}\right\|_{\beta, \sim}^{2}\right)\left(\frac{1}{r^{\alpha}}+\frac{1}{(t-r)^{\alpha^{\prime}}} r^{\alpha^{\prime}-\alpha}\right) .
\end{aligned}
$$

Since $\left|D_{t-}^{1-\alpha} \omega_{t-}[r]\right| \leq c(t-r)^{\beta^{\prime}+\alpha-1}\|\omega\|_{\beta^{\prime}}$, for $t>0$ we have

$$
\left|I_{1}(t)\right|_{V_{\gamma}} \leq c\|\omega\|_{\beta^{\prime}} t^{\beta^{\prime}}\left(1+\left\|u^{0}\right\|_{\beta, \sim}^{2}\right) \leq c\|\omega\|_{\beta^{\prime}} t^{\beta^{\prime}}\left(1+\left\|U^{0}\right\|_{W}^{2}\right)
$$

Similarly, we obtain

$$
\begin{aligned}
& \left\|D_{0+}^{2 \alpha-1}\left(S(t-\cdot) D G\left(u^{0}(\cdot)\right)\right)[r]\right\|_{L_{2}\left(V, V_{\gamma}\right)} \leq c\left(\frac{\left\|S(t-r) D G\left(u^{0}(r)\right)\right\|_{L_{2}\left(V, V_{\gamma}\right)}}{r^{2 \alpha-1}}\right. \\
& \quad+\int_{0}^{r} \frac{\left\|(S(t-r)-S(t-q)) D G\left(u^{0}(r)\right)\right\|_{L_{2}\left(V, V_{\gamma}\right)}}{(r-q)^{2 \alpha}} d q \\
& \left.\quad+\int_{0}^{r} \frac{\left\|S(t-q)\left(D G\left(u^{0}(r)\right)-D G\left(u^{0}(q)\right)\right)\right\|_{L_{2}\left(V, V_{\gamma}\right)}}{(r-q)^{2 \alpha}} d q\right) \\
& \leq c\left(\frac{c_{D G}}{r^{2 \alpha-1}}+c_{D G} \int_{0}^{r}(t-r)^{-\beta}(r-q)^{\beta-2 \alpha} d q+c_{D^{2} G}\left\|u^{0}\right\|_{\beta, \sim} \int_{0}^{r} \frac{(r-q)^{\beta} q^{-\beta}}{(r-q)^{2 \alpha}} d q\right) \\
& \leq c\left(r^{1-2 \alpha}+(t-r)^{-\beta} r^{\beta+1-2 \alpha}+\left\|u^{0}\right\|_{\beta, \sim} r^{1-2 \alpha}\right) .
\end{aligned}
$$
get

Since $\left\|D_{t-}^{1-\alpha} \mathcal{D}_{t-}^{1-\alpha} v^{0}[r]\right\| \leq c\left(\left\|v^{0}\right\|_{\beta+\beta^{\prime}, \sim}+\left\|u^{0}\right\|_{\beta, \sim}\|\omega\|_{\beta^{\prime}}\right) r^{-\beta}(t-r)^{\beta+\beta^{\prime}+2 \alpha-2}$, for $t>0$ we

$$
\left|I_{2}(t)\right|_{V_{\gamma}} \leq c t^{\beta^{\prime}}\left(1+\|\omega\|_{\beta^{\prime}}\right)\left(1+\left\|U^{0}\right\|_{W}^{2}\right)
$$

and this concludes the proof.

In the following, the pair $U^{0}=\left(u^{0}, v^{0}\right) \in \hat{W}_{0, T_{0}}$ is the (local) mild solution to $U=\mathcal{T}(U)$ obtained in Theorem 11. We denote $I_{0}=\left[0, T_{0}\right]$. 
5. Pathwise global solutions to SEEs driven by a Hölder path. In this section we want to go one step further and present the existence of a global solution to (2). The key fact of this result will be that we can build a global solution provided that the initial condition is regular enough. Starting with the initial condition $u_{0} \in V$, what we know according to Theorem 11, and in particular due to (18), is that the corresponding local solution to (2) satisfies $u^{0}\left(T_{0}\right) \in V_{\gamma}$. Therefore, in a second step we can pick precisely as initial condition $u^{0}\left(T_{0}\right)$ and construct the corresponding mild solution, which will turn out to be global thanks to a concatenation procedure which will be described below.

Throughout this section, we take $\hat{V}$ to be the space $V_{\gamma}$, where the condition on $\gamma$ will be detailed below in the next results.

To start with, we need to consider a new phase space: for any fixed $\omega \in C_{\beta^{\prime}}([0, T] ; V)$, let $\left(X_{0, T},\|\cdot\|_{X}\right)$ be the subspace of elements $U=(u, v)$ of the Banach space $C_{\beta}([0, T] ; V) \times$ $C_{\beta+\beta^{\prime}}\left(\Delta_{0, T} ; V \otimes V\right)$ such that the Chen equality (8) holds. The space $X_{0, T}$ has the norm

$$
\|U\|_{X}=\|u\|_{\beta}+\|v\|_{\beta+\beta^{\prime}}=\|u\|_{C}+\|U\|_{X}
$$

where

$$
\|U\|_{X}=\|u\|_{\beta}+\|v\|_{\beta+\beta^{\prime}}
$$

Let $\hat{X}_{0, T}$ be the (closed) subspace of $X_{0, T}$ with elements $U=(u, v)$ given by the limit points in $X_{0, T}$ of the set

$$
\begin{gathered}
\left\{\left(u_{n},\left(u_{n} \otimes \omega_{n}\right)\right): n \in \mathbb{N}, u_{n} \in C_{\gamma}([0, T] ; V), u_{n}(0) \in V_{\gamma},\right. \\
\left.\left(\omega_{n},\left(\omega_{n} \otimes_{S} \omega_{n}\right)\right) \text { satisfies }(\mathrm{H} 3)\right\},
\end{gathered}
$$

where $\alpha<\gamma$ (we recall that the parameter $\alpha$ appears in the definition of the fractional derivatives; see section 2). $\hat{X}_{0, T}$ is a complete metric space with the metric

$$
d_{\hat{X}}(U, \hat{U})=\|U-\hat{U}\|_{X}=\|u-\hat{u}\|_{\beta}+\|v-\hat{v}\|_{\beta+\beta^{\prime}}
$$

for $U, \hat{U} \in \hat{X}_{0, T}$. Moreover, elements of this space satisfy the Chen equality (8) with respect to $\omega$. Note that in the space $\hat{X}_{0, T}$ the additivity of the pathwise integral holds in the same sense as in Remark 8.

Consider now only elements of this space with fixed initial value $u(0)=u_{0} \in V_{\gamma}$; then we obtain a complete metric space $\hat{X}_{0, T, u_{0}}$ with metric

$$
d_{\hat{X}_{0, T}, u_{0}}(U, \hat{U})=\|U-\hat{U}\|_{X}=\|u-\hat{u}\|_{\beta}+\|v-\hat{v}\|_{\beta+\beta^{\prime}}
$$

We also use the notation $u_{0}$ to describe the constant function on $[0, T]$. Then $U_{0}:=\left(u_{0}, 0\right) \in$ $\hat{X}_{0, T, u_{0}}$ and

$$
d_{\hat{X}_{0, T}, u_{0}}\left(U, U_{0}\right)=\left\|U-U_{0}\right\|_{X}=\|u\|_{\beta}+\|v\|_{\beta+\beta^{\prime}}=\|U\|_{X} \cdot
$$

In particular, a closed ball in $\hat{X}_{0, T, u_{0}}$ with center $U_{0}$ and radius $R$ is given by

$$
B_{\hat{X}_{0, T}, u_{0}}(R)=\left\{U=(u, v) \in \hat{X}_{0, T, u_{0}}:\|u\|_{\beta}+\|v\|_{\beta+\beta^{\prime}}=\|U\|_{X} \leq R\right\} .
$$

Copyright $\odot$ by SIAM. Unauthorized reproduction of this article is prohibited. 
In a similar manner we can define qualitatively the spaces $\hat{X}_{\check{T}, \hat{T}, \check{u}}$ over intervals $[\check{T}, \hat{T}]$ and with respect to elements $\check{U}=(\check{u}, 0)$, with $\check{u} \in V_{\gamma}$.

The following two lemmas will play an important role to prove the existence of a global solution to (2) provided that the initial condition belongs to an adequate space $V_{\gamma}$. Their proofs are omitted since they are quite similar to the corresponding results when having an initial condition in $V$ and $U \in \hat{W}_{0, T}$; see [11].

Lemma 12. Suppose $(\mathrm{H})$ holds. Then for any $1 \geq \gamma \geq \beta$ and any $T>0$ there exists $c>0$ such that for $u_{0} \in V_{\gamma}$ and $U \in \hat{X}_{0, T, u_{0}}$ we have

$$
\begin{aligned}
\|\mathcal{T}(U)\|_{X} & \leq c\left(T^{\beta^{\prime}-\beta}\left(1+T^{2 \beta}\|U\|_{X}^{2}\right)+T^{\gamma-\beta}\left|u_{0}\right|_{V_{\gamma}}\right), \\
\left|\mathcal{T}_{1}(U)(T)\right|_{V_{\gamma}} & \leq c T^{\beta^{\prime}}\left(1+T^{2 \beta}\|U\|_{X}^{2}\right)+\left|u_{0}\right|_{V_{\gamma}} .
\end{aligned}
$$

The constant $c$ depends on $T,\|\omega\|_{\beta^{\prime}}$, and $\left\|\left(\omega \otimes_{S} \omega\right)\right\|_{2 \beta^{\prime}}$.

Let us only point out that by Lemma 1 , the map $t \mapsto S(t) u_{0}$ is $\beta$-Hölder-continuous, which is necessary to estimate the $X$-norm of $\mathcal{T}$. More precisely, we have

$$
\left\|S(\cdot) u_{0}\right\|_{\beta} \leq T^{\gamma-\beta}\left|u_{0}\right|_{V_{\gamma}} .
$$

Lemma 13. Suppose $(\mathrm{H})$ holds.

(i) Then for any $1 \geq \gamma \geq \beta$ and any $T>0$ there exists $c>0$ such that for $\tilde{U}=(\tilde{u}, \tilde{v}), \hat{U}=$ $(\hat{u}, \hat{v}) \in \hat{X}_{0, T}$ with $\tilde{u}(0)=\tilde{u}_{0} \in V_{\gamma}, \hat{u}(0)=\hat{u}_{0} \in V_{\gamma}$, we have

$$
\begin{aligned}
\|\mathcal{T}(\tilde{U})-\mathcal{T}(\hat{U})\| \|_{X} & \leq c T^{\beta^{\prime}-\beta}\left(1+T^{2 \beta}\left(\|\tilde{U}\|_{X}^{2}+\|\hat{U}\|_{X}^{2}\right)\right)\left(\left|\tilde{u}_{0}-\hat{u}_{0}\right|_{V_{\gamma}}\right. \\
& \left.+\|\tilde{U}-\hat{U}\|_{X}\right)+T^{\gamma-\beta}\left|\tilde{u}_{0}-\hat{u}_{0}\right|_{V_{\gamma}}
\end{aligned}
$$

and

$$
\begin{aligned}
\left|\mathcal{T}_{1}(\tilde{U})(T)-\mathcal{T}_{1}(\hat{U})(T)\right|_{V_{\gamma}} \leq & c T^{\beta^{\prime}}\left(1+T^{2 \beta}\left(\||\tilde{U}|\|_{X}^{2}+\|\hat{U}\|_{X}^{2}\right)\right)\left(\left|\tilde{u}_{0}-\hat{u}_{0}\right|_{V_{\gamma}}\right. \\
& \left.+\|\tilde{U}-\hat{U}\| \|_{X}\right)+c\left|\tilde{u}_{0}-\hat{u}_{0}\right|_{V_{\gamma}} .
\end{aligned}
$$

(ii) If $U \in \hat{X}_{0, T, u_{0}}$ we get

$$
\lim _{n \rightarrow \infty}\left\|\mathcal{T}\left(U, \omega_{n},\left(\omega_{n} \otimes_{S} \omega_{n}\right), u_{0}\right)-\mathcal{T}\left(U, \omega,\left(\omega \otimes_{S} \omega\right), u_{0}\right)\right\|_{X}=0 .
$$

This convergence is uniform for $U \in \hat{X}_{0, T, u_{0}}$ contained in a bounded set.

Proof. We only give an idea of the proof of the first inequality of (i), which is based on the different estimates for $G$ coming from Lemma 2 .

First of all, denoting $\Delta u=\tilde{u}-\hat{u}$,

$$
\begin{aligned}
& \|G(\tilde{u}(r))-G(\hat{u}(r))\|_{L_{2}\left(V, V_{\gamma}\right)} \leq c_{D G}\left(\left|\tilde{u}(r)-\tilde{u}_{0}-\hat{u}(r)+\hat{u}_{0}\right|+\left|\tilde{u}_{0}-\hat{u}_{0}\right|\right) \\
& \leq c_{D G}\left(\sup _{0 \leq q<r \leq T} \frac{|\tilde{u}(r)-\hat{u}(r)-(\tilde{u}(q)-\hat{u}(q))|}{(r-q)^{\beta}} T^{\beta}+\left|\tilde{u}_{0}-\hat{u}_{0}\right|\right) \\
& =c_{D G}\left(\|\Delta u\|_{\beta} T^{\beta}+\left|\tilde{u}_{0}-\hat{u}_{0}\right|\right) \leq c c_{D G}\left(\|\Delta u\|_{\beta} T^{\beta}+\left|\tilde{u}_{0}-\hat{u}_{0}\right|_{V_{\gamma}}\right)
\end{aligned}
$$

Copyright (C) by SIAM. Unauthorized reproduction of this article is prohibited. 
and similarly for $D G(u(r))$. Note that the last inequality above is a consequence of the continuous embedding $V_{\gamma} \subset V$. Moreover, thanks to Lemma 2 we get

$$
\begin{aligned}
& \|D G(\tilde{u}(r))-D G(\hat{u}(r))-(D G(\tilde{u}(q))-D G(\hat{u}(q)))\|_{L_{2}\left(V, V_{\gamma}\right)} \\
\leq & c_{D^{2} G}\|\Delta u\|_{\beta}(r-q)^{\beta}+c c_{D^{3} G}\left(\|\Delta u\|_{\beta} T^{\beta}+\left|\tilde{u}_{0}-\hat{u}_{0}\right|_{V_{\gamma}}\right)\left(\|\tilde{u}\|_{\beta}+\|\hat{u}\|_{\beta}\right)(r-q)^{\beta}
\end{aligned}
$$

and

$$
\begin{aligned}
\| G(\tilde{u}(r)) & -G(\tilde{u}(q))-D G(\tilde{u}(q))(\tilde{u}(r)-\tilde{u}(q)) \\
& -(G(\hat{u}(r))-G(\hat{u}(q))-D G(\hat{u}(q))(\hat{u}(r)-\hat{u}(q))) \|_{L_{2}\left(V, V_{\gamma}\right)} \\
& \leq c_{D^{2} G}\left(\|\tilde{u}\|_{\beta}+\|\hat{u}\|_{\beta}\right)\|\Delta u\|_{\beta}(r-q)^{2 \beta} \\
& +c c_{D^{3} G}\|\hat{u}\|_{\beta}\left(\|\Delta u\|_{\beta} T^{\beta}+\left|\tilde{u}_{0}-\hat{u}_{0}\right|_{V_{\gamma}}\right)\left(2\|\tilde{u}\|_{\beta}+\|\hat{u}\|_{\beta}\right)(r-q)^{2 \beta} .
\end{aligned}
$$

In particular, when $\tilde{U}, \hat{U} \in \hat{X}_{0, T, u_{0}}$, the first inequality of Lemma 13(i) becomes

$$
\|\mathcal{T}(\tilde{U})-\mathcal{T}(\hat{U})\|_{X} \leq c T^{\beta^{\prime}-\beta}\left(1+T^{2 \beta}\left(\|\tilde{U}\|_{X}^{2}+\|\hat{U}\|_{X}^{2}\right)\right)\|\tilde{U}-\hat{U}\|_{X}
$$

We can also consider $\mathcal{T}$ on other time intervals, that is, defined on intervals $[s, T]$, where $s>0$. Indeed, exchanging $\hat{X}_{0, T}$ by $\hat{X}_{s, T}$ these operators satisfy qualitatively the same estimates as $\mathcal{T}$ stated in Lemmas 12 and 13. As we already said in section 3, we denote these operators by $\mathcal{T}^{s}$.

In what follows we describe how to construct a global solution to our equation on $[0, T]$.

In a first step, we shall focus on proving the existence of a solution belonging to $\hat{X}_{T_{0}, T, u_{0}^{1}}$, where $T_{0}$ was defined in Theorem 11 . We would like to mention that even though the above estimates of $\mathcal{T}$ contain quadratic expressions, it is possible to construct solutions defined in adequate small time intervals $\left[T_{i-1}, T_{i}\right]$, namely, solutions belonging to $\hat{X}_{T_{i-1}, T_{i}, u^{i-1}\left(T_{i-1}\right)}$ for suitable initial conditions, to further these solutions being joined together to form a whole solution in $\hat{X}_{T_{0}, T, u_{0}^{1}}$. The choice of the starting point in each step will be clear in the construction of the solution. To prove the existence of these solutions we apply Lemmas 12 and 13 on every interval $\left[T_{i-1}, T_{i}\right]$. Note that the appearing constant $c$ in these lemmas depends on those intervals, on the $\beta^{\prime}$-norm of $\omega$ in $\left[T_{i-1}, T_{i}\right]$ and on the $2 \beta^{\prime}$-norm of $\left(\omega \otimes_{S} \omega\right)$ with respect to $\bar{\Delta}_{T_{i-1}, T_{i}}$. However, the following results show that we can choose a larger constant independent of these intervals and triangles, and only depending on the given number $T$ and the norms of $\omega,\left(\omega \otimes_{S} \omega\right)$ with respect to $[0, T], \bar{\Delta}_{0, T}$. In addition, $c$ will be chosen large enough so that we also take the construction of the local solution given in Theorem 11 into account.

The way in which we join together these pieces of solutions is described in general as follows.

Definition 14. For $0<a<b<c$ (resp., $0 \leq a<b<c$ ) we consider the concatenation $U=(u, v)$ of elements $U^{1}=\left(u^{1}, v^{1}\right) \in \hat{X}_{a, b}$ with $u^{1}(a) \in V_{\gamma}\left(\right.$ or $U^{1}=\left(u^{1}, v^{1}\right) \in \hat{W}_{a, b}$ with 
$\left.u^{1}(a) \in V\right)$ and $U^{2}=\left(u^{2}, v^{2}\right) \in \hat{X}_{b, c}$, with $u^{2}(b)=u^{1}(b)$, defined by

$$
\begin{aligned}
& u(t)= \begin{cases}u^{1}(t) & : \quad a \leq t \leq b, \\
u^{2}(t) & : \quad b \leq t \leq c\end{cases} \\
& v(s, t)=\left\{\begin{array}{lr}
v^{1}(s, t) & : a \leq s \leq t \leq b, \\
v^{2}(s, t) & : \quad b \leq s \leq t \leq c, \\
\left(u^{1}(b)-u^{1}(s)\right) \otimes_{V}(\omega(t)-\omega(b))+v^{1}(s, b)+v^{2}(b, t) & : \quad s \leq b<t .
\end{array}\right.
\end{aligned}
$$

Note that in the previous definition $v$ has been defined according to the Chen equality. It is straightforward to check that $U$ satisfies the Chen equality and $U \in \hat{X}_{a, c}$ (or $U \in \hat{W}_{a, c}$ when $U^{1} \in \hat{W}_{a, b}$ with $\left.u^{1}(a) \in V\right)$. For a proof of a similar statement see Lemma 18 below.

In the next result we present the existence and uniqueness of a first piece of solution $U^{1}=\left(u^{1}, v^{1}\right) \in \hat{X}_{T_{0}, T_{1}, u_{0}^{1}}$ with initial condition $u_{0}^{1} \in V_{\gamma}$.

Later on, we will take $u_{0}^{1}=u^{0}\left(T_{0}\right)$, where we recall that $U^{0}=\left(u^{0}, v^{0}\right)$ denotes the solution to $(2)$ in $\hat{W}_{0, T_{0}}$ with initial condition $u_{0} \in V$; see Theorem 11.

Theorem 15. Suppose $(\mathrm{H})$ holds and that $\gamma+\beta^{\prime}>1, \gamma \leq 1$. Then for any $u_{0}^{1} \in V_{\gamma}$ there exists a small enough $T_{1}>T_{0}$ depending on the data such that $U=\mathcal{T}^{T_{0}}(U)$ has a unique mild solution $U^{1}=\left(u^{1}, v^{1}\right) \in \hat{X}_{T_{0}, T_{1}, u_{0}^{1}}$.

Proof. Define $\left|u_{0}^{1}\right|_{V_{\gamma}}=: \rho_{0}$. Let us consider $K\left(\rho_{0}\right) \geq 1$ such that for $K \geq K\left(\rho_{0}\right)$, the following relations are fulfilled:

$$
\begin{aligned}
& \rho_{0}+2 c K^{-\beta^{\prime}}<K^{1-\beta^{\prime}}, \\
& 4 c^{2} K^{-\beta^{\prime}-\beta}\left(K^{\beta-\gamma} K^{1-\beta^{\prime}}+K^{\beta-\beta^{\prime}}\right)<1, \\
& c K^{\beta-\beta^{\prime}}\left(1+2 K^{-2 \beta}\left(8 c^{2} K^{2 \beta-2 \gamma} K^{2-2 \beta^{\prime}}+8 c^{2} K^{2 \beta-2 \beta^{\prime}}\right)\right)<\frac{1}{2}, \\
& c K^{-\beta^{\prime}}+c K^{-\beta^{\prime}-2 \beta}\left(8 c^{2} K^{2 \beta-2 \gamma} K^{2-2 \beta^{\prime}}+8 c^{2} K^{2 \beta-2 \beta^{\prime}}\right)<2 c K^{-\beta^{\prime}} .
\end{aligned}
$$

Note that $\rho_{0}<K^{1-\beta^{\prime}}$ and that the last three inequalities are true due to the assumption $\gamma+\beta^{\prime}>1$ and (H1).

In what follows we prove that condition (23) ensures that $\mathcal{T}^{T_{0}}$ maps a ball $B_{\hat{X}_{T_{0}, T_{1}, u_{0}^{1}}}\left(R_{1}\right)$ into itself and it is a contraction on it, for $T_{0}$ given in Theorem 11, for a small enough $T_{1}$, and for an appropriate radius $R_{1}$. In particular, let us take $T_{1}=T_{0}+\Delta T_{1}$, where $\Delta T_{1}=K^{-1}$. Then, by Lemma 12 we have

$$
\|\mathcal{T}(U)\|_{X_{T_{0}, T_{1}}} \leq c\left(\Delta T_{1}^{\gamma-\beta} \rho_{0}+\Delta T_{1}^{\beta^{\prime}-\beta}+\Delta T_{1}^{\beta^{\prime}+\beta}\|U\|_{X_{T_{0}, T_{1}}}^{2}\right)
$$

Hence, to find a ball $B_{\hat{X}_{T_{0}, T_{1}, u_{0}^{1}}}\left(R_{1}\right)$ that will be mapped into itself we calculate the minor root $R_{1}$ of the quadratic equation

$$
x=c\left(\Delta T_{1}^{\gamma-\beta} \rho_{0}+\Delta T_{1}^{\beta^{\prime}-\beta}+\Delta T_{1}^{\beta^{\prime}+\beta} x^{2}\right)
$$

Copyright (C) by SIAM. Unauthorized reproduction of this article is prohibited. 
which is given by

$$
R_{1}:=\frac{2 c\left(\Delta T_{1}^{\gamma-\beta} \rho_{0}+\Delta T_{1}^{\beta^{\prime}-\beta}\right)}{1+\sqrt{1-4 c^{2} \Delta T_{1}^{\beta^{\prime}+\beta}\left(\Delta T_{1}^{\gamma-\beta} \rho_{0}+\Delta T_{1}^{\beta^{\prime}-\beta}\right)}}<2 c\left(\Delta T_{1}^{\gamma-\beta} \rho_{0}+\Delta T_{1}^{\beta^{\prime}-\beta}\right) .
$$

This root is well-defined (indeed it is a real root) due to the definition of $\Delta T_{1}$ and the first and second inequalities of (23), since these conditions in particular imply that

$$
1-4 c^{2} \Delta T_{1}^{\beta^{\prime}+\beta}\left(\Delta T_{1}^{\gamma-\beta} \rho_{0}+\Delta T_{1}^{\beta^{\prime}-\beta}\right)>0 .
$$

Note that if $U \in B_{X_{T_{0}, T_{1}, u_{0}^{1}}}\left(R_{1}\right)$, then

$$
\|U\|_{X_{T_{0}, T_{1}}}^{2} \leq R_{1}^{2} \leq 8 c^{2} \Delta T_{1}^{2 \gamma-2 \beta} \rho_{0}^{2}+8 c^{2} \Delta T_{1}^{2 \beta^{\prime}-2 \beta},
$$

and thanks to $(22)$ we can claim that $\mathcal{T}$ is a contraction on $B_{\hat{X}_{T_{0}, T_{1}, u_{0}^{1}}}\left(R_{1}\right)$ if

$$
c \Delta T_{1}^{\beta^{\prime}-\beta}\left(1+2 \Delta T_{1}^{2 \beta}\left(8 c^{2} \Delta T_{1}^{2 \gamma-2 \beta} \rho_{0}^{2}+8 c^{2} \Delta T_{1}^{2 \beta^{\prime}-2 \beta}\right)\right)<\frac{1}{2} .
$$

The previous relation follows from the first and third inequalities of (23).

To see that $\mathcal{T}\left(B_{\hat{X}_{T_{0}, T_{1}, u_{0}^{1}}}\right)\left(R_{1}\right) \in \hat{X}_{T_{0}, T_{1}, u_{0}^{1}}$ we approximate an element $U=(u, v) \in$ $B_{\hat{X}_{T_{0}, T_{1}, u_{0}^{1}}}\left(R_{1}\right)$ by a sequence $\left(u_{n},\left(u_{n} \otimes \omega_{n}\right)\right)$ from $(20)$. We note that $\mathcal{T}_{1}\left(\left(u_{n},\left(u_{n} \otimes \omega_{n}\right)\right), \omega_{n}\right.$, $\left.u_{n}(0)\right)(0)=u_{n}(0) \in V_{\gamma}$ and $\mathcal{T}_{1}\left(\left(u_{n},\left(u_{n} \otimes \omega_{n}\right)\right), \omega_{n}, u_{n}(0)\right) \in C_{\gamma}([0, T] ; V)$ for any $\gamma \in(0,1)$; see Pazy [22] Theorem 4.3.1. Therefore $\mathcal{T}_{2}\left(\left(u_{n},\left(u_{n} \otimes \omega_{n}\right)\right), \omega_{n},\left(\omega_{n} \otimes_{S} \omega_{n}\right), u_{n}(0)\right)$ can be defined as $\left(\mathcal{T}_{1}\left(\left(u_{n},\left(u_{n} \otimes \omega_{n}\right)\right), \omega_{n}, u_{n}(0)\right) \otimes \omega_{n}\right)$ given by (10). The previous considerations mean that $\mathcal{T}\left(\left(u_{n},\left(u_{n} \otimes \omega_{n}\right)\right), \omega_{n},\left(\omega_{n} \otimes_{S} \omega_{n}\right), u_{n}(0)\right)$ belongs to the set $(20)$. Now it suffices to use the first inequality of (i) and (ii) in Lemma 13 to ensure that $\mathcal{T}\left(U, \omega,\left(\omega \otimes_{S} \omega\right), u_{0}\right) \in \hat{X}_{T_{0}, T_{1}, u_{0}^{1}}$.

Therefore, we obtain that $\mathcal{T}^{T_{0}}$ has a fixed point $U^{1}=\left(u^{1}, v^{1}\right) \in B_{\hat{X}_{T_{0}, T_{1}, u_{0}^{1}}}\left(R_{1}\right)$. Furthermore, by Lemma 12 and the first and last inequalities of (23), we get

$$
\begin{aligned}
\left|u^{1}\left(T_{1}\right)\right|_{V_{\gamma}} & \leq c \Delta T_{1}^{\beta^{\prime}}\left(1+\Delta T_{1}^{2 \beta}\left\|U^{1}\right\|_{X_{T_{0}, T_{1}}}^{2}\right)+\left|u_{0}^{1}\right|_{V_{\gamma}} \\
& \leq c \Delta T_{1}^{\beta^{\prime}}+c \Delta T_{1}^{\beta^{\prime}+2 \beta}\left(8 c^{2} \Delta T_{1}^{2 \gamma-2 \beta} \rho_{0}^{2}+8 c^{2} \Delta T_{1}^{2 \beta^{\prime}-2 \beta}\right)+\rho_{0} \\
& <2 c \Delta T_{1}^{\beta^{\prime}}+\rho_{0}<K^{1-\beta^{\prime}} .
\end{aligned}
$$

We point out that in the previous result it was crucial to use the seminorm $\|\cdot\|_{X}$ instead of the norm $\|\cdot\|_{X}$. If we had used directly the norm, then we would not have found an appropriate $K$ fulfilling (23) since we cannot make the $C$-norm small if the interval $\left[T_{0}, T_{1}\right]$ (or in general any interval $\left[T_{i-1}, T_{i}\right]$ ) is small.

So far we have obtained the existence of a local solution $U^{1}=\left(u^{1}, v^{1}\right) \in \hat{X}_{T_{0}, T_{1}, u_{0}^{1}}$ with initial condition $u_{0}^{1} \in V_{\gamma}$, that is, we have found a solution whose path component $u^{1}$ is defined 
on the interval $I_{1}=\left[T_{0}, T_{1}\right]$ for $T_{1}=T_{0}+\frac{1}{K}$. Now we would like to repeat the same arguments in Theorem 15 in time intervals

$$
I_{2}=\left[T_{1}, T_{2}\right]:=\left[T_{1}, T_{1}+\frac{1}{2 K}\right], \quad \cdots \cdots \quad I_{i}:=\left[T_{i-1}, T_{i}\right]=\left[T_{i-1}, T_{i-1}+\frac{1}{i K}\right]
$$

for $i=2, \ldots, i^{*}$ for appropriate $i^{*}$; see Theorem 16 .

Theorem 16. Let $T>0$ be some number. Suppose that $(\mathrm{H})$ holds and $\gamma+\beta^{\prime}>1, \gamma \leq 1$. Suppose also that $u_{0}^{1} \in V_{\gamma}$ is given by $u^{0}\left(T_{0}\right)=\mathcal{T}_{1}\left(\left(u^{0}, v^{0}\right), \omega, u_{0}\right)\left(T_{0}\right), u_{0} \in V$ see Theorem 11. Then there exist $i^{*}$ intervals $\left[T_{0}, T_{1}\right], \cdots\left[T_{i^{*}-1}, T_{i^{*}}\right]$ where $T_{i^{*}-1}<T \leq T_{i^{*}}$ such that on any of these intervals

$$
U^{i}=\mathcal{T}^{T_{i-1}}\left(U^{i}, \omega,\left(\omega \otimes_{S} \omega\right), u^{i-1}\left(T_{i-1}\right)\right)
$$

has a unique solution $U^{i}=\left(u^{i}, v^{i}\right) \in \hat{X}_{T_{i-1}, T_{i}, u^{i-1}\left(T_{i-1}\right)}$. Therefore, each of these local solutions can be approximated by the classical solutions $U_{n}^{i}=\left(u_{n}^{i},\left(u_{n}^{i} \otimes \omega_{n}\right)\right)$ that satisfy

$$
U_{n}^{i}=\mathcal{T}^{T_{i-1}}\left(U_{n}^{i}, \omega_{n},\left(\omega_{n} \otimes_{S} \omega_{n}\right), u_{n}^{i-1}\left(T_{i-1}\right)\right) .
$$

Proof. The proof consists of applying an induction procedure, with the starting step given in the proof of Theorem 15. In order to do that, assume the following generalization of the assumption (23): $K\left(\rho_{0}\right) \geq 1$ is such that for $K \geq K\left(\rho_{0}\right)$ and $i \in \mathbb{N}$ the following inequalities are fulfilled:

$$
\begin{aligned}
\rho_{0} & +\sum_{j=1}^{i} 2 c(K j)^{-\beta^{\prime}} \leq \rho_{0}+2 c K^{-\beta^{\prime}} \frac{1}{1-\beta^{\prime}} i^{1-\beta^{\prime}}<(K i)^{1-\beta^{\prime}}, \\
& 4 c^{2}(K i)^{-\beta^{\prime}-\beta}\left((K i)^{\beta-\gamma}(K i)^{1-\beta^{\prime}}+(K i)^{\beta-\beta^{\prime}}\right)<1, \\
& c(K i)^{\beta-\beta^{\prime}}\left(1+2(K i)^{-2 \beta}\left(8 c^{2}(K i)^{2 \beta-2 \gamma}(K i)^{2-2 \beta^{\prime}}+8 c^{2}(K i)^{2 \beta-2 \beta^{\prime}}\right)\right)<\frac{1}{2}, \\
& c(K i)^{-\beta^{\prime}}+c(K i)^{-\beta^{\prime}-2 \beta}\left(8 c^{2}(K i)^{2 \beta-2 \gamma}(K i)^{2-2 \beta^{\prime}}+8 c^{2}(K i)^{2 \beta-2 \beta^{\prime}}\right) \\
& <2 c(K i)^{-\beta^{\prime}} .
\end{aligned}
$$

Once again, we stress that these inequalities hold due to the condition $\gamma+\beta^{\prime}>1$.

Assume that we have obtained pieces of local solutions $U^{1}=\left(u^{1}, v^{1}\right) \in \hat{X}_{T_{0}, T_{1}, u^{0}\left(T_{0}\right)}, \ldots$, $U^{i-1}=\left(u^{i-1}, v^{i-1}\right) \in \hat{X}_{T_{i-2}, T_{i-1}, u^{i-2}\left(T_{i-2}\right)}$ such that

$$
\left|u^{i-1}\left(T_{i-1}\right)\right|_{V_{\gamma}}<\rho_{0}+\sum_{j=1}^{i-1} 2 c(K j)^{-\beta^{\prime}}
$$

for $i=2,3, \ldots$, and $T_{i-1}<T$. Note that (27) is nothing but the generalization of the property (25). Now we give some details on how to obtain the next piece of local solution $U^{i}=\left(u^{i}, v^{i}\right)$ : we set $T_{i}=T_{i-1}+\Delta T_{i}, \Delta T_{i}=(K i)^{-1}$ if $T_{i}<T$, and $T_{i}=T$ in the other cases. Then, by the first inequality of (26) we know that $\left|u^{i-1}\left(T_{i-1}\right)\right|_{V_{\gamma}}<(K i)^{1-\beta^{\prime}}$. Following the steps of Theorem 15, (26) together with the Banach fixed point theorem ensures the existence of a

Copyright (C) by SIAM. Unauthorized reproduction of this article is prohibited. 
local solution $U^{i}=\left(u^{i}, v^{i}\right) \in B_{\hat{X}_{T_{i-1}, T_{i}, u^{i-1}\left(T_{i-1}\right)}}\left(R_{i}\right)$ which is unique, where $R_{i}$ is the minor root of (24) when replacing $\rho_{0}$ by $(K i)^{1-\beta^{\prime}}$ and $\Delta T_{1}$ by $\Delta T_{i}$. In addition, (27) and the first inequality and the last one of (26) imply

$$
\begin{aligned}
\left|u^{i}\left(T_{i}\right)\right|_{V_{\gamma}} & \leq c \Delta T_{i}^{\beta^{\prime}}\left(1+\Delta T_{i}^{2 \beta}\|U\|_{X_{T_{i-1}, T_{i}}}^{2}\right)+\left|u^{i-1}\left(T_{i-1}\right)\right|_{V_{\gamma}} \\
& \leq c \Delta T_{i}^{\beta^{\prime}}+c \Delta T_{i}^{\beta^{\prime}+2 \beta}\left(8 c^{2} \Delta T_{i}^{2 \gamma-2 \beta} \Delta T_{i}^{2 \beta^{\prime}-2}+8 c^{2} \Delta T_{i}^{2 \beta^{\prime}-2 \beta}\right)+\left|u^{i-1}\left(T_{i-1}\right)\right|_{V_{\gamma}} \\
& <2 c(K i)^{-\beta^{\prime}}+\rho_{0}+\sum_{j=1}^{i-1} 2 c(K j)^{-\beta^{\prime}}=\sum_{j=1}^{i} 2 c(K j)^{-\beta^{\prime}}+\rho_{0}<(K i)^{1-\beta^{\prime}} .
\end{aligned}
$$

Since $\sum_{i}(K i)^{-1}=\infty$ there is an $i^{*} \in \mathbb{N}$ such that $T_{i^{*}} \wedge T=T$.

Finally for the convergence observe that

$$
\begin{aligned}
& \left\|U_{n}^{i}-U^{i}\right\|_{X} \\
= & \left\|\mathcal{T}^{T_{i-1}}\left(U_{n}^{i}, \omega_{n},\left(\omega_{n} \otimes_{S} \omega_{n}\right), u_{n}^{i-1}\left(T_{i-1}\right)\right)-\mathcal{T}^{T_{i-1}}\left(U^{i}, \omega,\left(\omega \otimes_{S} \omega\right), u^{i-1}\left(T_{i-1}\right)\right)\right\|_{X} \\
\leq & \left\|\mathcal{T}^{T_{i-1}}\left(U_{n}^{i}, \omega_{n},\left(\omega_{n} \otimes_{S} \omega_{n}\right), u_{n}^{i-1}\left(T_{i-1}\right)\right)-\mathcal{T}^{T_{i-1}}\left(U_{n}^{i}, \omega_{n},\left(\omega_{n} \otimes_{S} \omega_{n}\right), u^{i-1}\left(T_{i-1}\right)\right)\right\|_{X} \\
+ & \left\|\mathcal{T}^{T_{i-1}}\left(U_{n}^{i}, \omega_{n},\left(\omega_{n} \otimes_{S} \omega_{n}\right), u^{i-1}\left(T_{i-1}\right)\right)-\mathcal{T}^{T_{i-1}}\left(U_{n}^{i}, \omega,\left(\omega \otimes_{S} \omega\right), u^{i-1}\left(T_{i-1}\right)\right)\right\| \|_{X} \\
+ & \left\|\mathcal{T}^{T_{i-1}}\left(U_{n}^{i}, \omega,\left(\omega \otimes_{S} \omega\right), u^{i-1}\left(T_{i-1}\right)\right)-\mathcal{T}^{T_{i-1}}\left(U^{i}, \omega,\left(\omega \otimes_{S} \omega\right), u^{i-1}\left(T_{i-1}\right)\right)\right\|_{X} .
\end{aligned}
$$

Now we can apply Lemma 13 to estimate these terms:

$$
\begin{aligned}
& \left\|U_{n}^{i}-U^{i}\right\|_{X} \leq c \Delta T_{i}^{\beta^{\prime}-\beta}\left(1+2 \Delta T_{i}^{2 \beta}\left\|U_{n}^{i}\right\|_{X}^{2}\right)\left|u_{n}^{i-1}\left(T_{i-1}\right)-u^{i-1}\left(T_{i-1}\right)\right|_{V_{\gamma}} \\
+ & c \Delta T_{i}^{\gamma-\beta}\left|u_{n}^{i-1}\left(T_{i-1}\right)-u^{i-1}\left(T_{i-1}\right)\right|_{V_{\gamma}} \\
+ & \left\|\mathcal{T}^{T_{i-1}}\left(U_{n}^{i}, \omega_{n},\left(\omega_{n} \otimes_{S} \omega_{n}\right), u^{i-1}\left(T_{i-1}\right)\right)-\mathcal{T}^{T_{i-1}}\left(U_{n}^{i}, \omega,\left(\omega \otimes_{S} \omega\right), u^{i-1}\left(T_{i-1}\right)\right)\right\|_{X} \\
+ & c \Delta T_{i}^{\beta^{\prime}-\beta}\left(1+\Delta T_{i}^{2 \beta}\left(\left\|U_{n}^{i}\right\|_{X}^{2}+\left\|U^{i}\right\|_{X}^{2}\right)\right)\left\|U_{n}^{i}-U^{i}\right\|_{X} .
\end{aligned}
$$

For the solution $U^{i-1}$ on the interval $\left[T_{i-2}, T_{i-1}\right]$ we can assume that $\mid u_{n}^{i-1}\left(T_{i-1}\right)-u^{i-1}\left(T_{i-1}\right)$ $\mid V_{\gamma}$ tends to zero, which gives the convergence of the first and second terms. This is also true for $U^{0}$ considered in section 4; see (19). The convergence of the third expression follows by Lemma 13(ii). For the last expression, we mention that $U_{n}^{i}$ satisfies similar a priori estimates as $U^{i}$, which follow by Lemma 12. In particular, thanks to (H3) the constant $c$ for $U_{n}^{i}$ which depends on $\omega_{n},\left(\omega_{n} \otimes_{S} \omega_{n}\right)$ can be chosen very close to the constant for $U^{i}$, and therefore $\left\|U_{n}^{i}\right\|_{X}$ is close to $\left\|U^{i}\right\|_{X}$ such that

$$
c \Delta T_{i}^{\beta^{\prime}-\beta}\left(1+\Delta T_{i}^{2 \beta}\left(\left\|U_{n}^{i}\right\|_{X}^{2}+\left\|U^{i}\right\|_{X}^{2}\right)\right) \leq q,
$$

where $q<1$. Summarizing we find the desired convergence, since $\left\|U_{n}^{i}-U^{i}\right\|_{X}$ is equivalent to

$$
\left|u_{n}^{i}\left(T_{i-1}\right)-u^{i}\left(T_{i-1}\right)\right|+\left\|U_{n}^{i}-U^{i}\right\|_{X},
$$

which can be estimated by $\left|u_{n}^{i}\left(T_{i-1}\right)-u^{i}\left(T_{i-1}\right)\right|_{V_{\gamma}}+\left\|U_{n}^{i}-U^{i}\right\|_{X}$, and this sum converges to zero when $n \rightarrow \infty$. 
Then we can prove the following result.

Lemma 17. The iterated concatenation $U=(u, v)$ of $U^{i}=\left(u^{i}, v^{i}\right)$ for $i=0,1, \ldots, i^{*}$ (in the sense of Definition 14) is such that $U \in C_{\beta, \sim}([0, T] ; V) \times C_{\beta+\beta^{\prime}, \sim}\left(\Delta_{0, T} ; V \otimes V\right)$.

Proof. Given $(s, t) \in \Delta_{0, T}$ we know that

$$
T_{i_{s}-1} \leq s \leq T_{i_{s}}<\cdots<T_{i_{t}-1}<t \leq T_{i_{t}}
$$

where $I_{t_{i_{s}}}=\left[T_{i_{s}-1}, T_{i_{s}}\right]$ and so on. By an iterated application of the Chen equality we get

$$
\begin{aligned}
v(s, t)= & v^{i_{s}}\left(s, T_{i_{s}}\right)+v^{i_{s}+1}\left(T_{i_{s}}, T_{i_{s}+1}\right)+\cdots+v^{i_{t}}\left(T_{i_{t}-1}, t\right) \\
& +\left(u^{i_{s}}\left(T_{i_{s}}\right)-u^{i_{s}}(s)\right) \otimes_{V}\left(\omega(t)-\omega\left(T_{i_{s}}\right)\right) \\
& +\left(u^{i_{s}+1}\left(T_{i_{s}+1}\right)-u^{i_{s}+1}\left(T_{i_{s}}\right)\right) \otimes_{V}\left(\omega(t)-\omega\left(T_{i_{s}+1}\right)\right) \\
& \vdots \\
& +\left(u^{i_{t}-1}\left(T_{i_{t}-1}\right)-u^{i_{t}-1}\left(T_{i_{t}-2}\right)\right) \otimes_{V}\left(\omega(t)-\omega\left(T_{i_{t}-1}\right)\right) .
\end{aligned}
$$

Now taking the $V \otimes V$-norm, applying the triangle inequality, multiplying the expression by $s^{\beta} /(t-s)^{\beta+\beta^{\prime}}$, and taking the supremum for $0<s<t<T$ we obtain

$$
\begin{aligned}
\|v\|_{\beta+\beta^{\prime}, \sim} \leq & \left\|v^{0}\right\|_{\beta+\beta^{\prime}, \sim, \Delta_{I_{0}}}+\left\|u^{0}\right\|_{\beta, \sim, I_{0}}\|\omega\|_{\beta^{\prime},[0, T]} \\
& +T^{\beta} \sum_{i=1}^{i^{*}}\left\|v^{i}\right\|_{\beta+\beta^{\prime}, \bar{\Delta}_{I_{i}}}+T^{\beta} \sum_{i=1}^{i^{*}-1}\left\|u^{i}\right\|_{\beta, I_{i}}\|\omega\|_{\beta^{\prime},[0, T]}<\infty .
\end{aligned}
$$

Moreover, for the first component of $U$ we get

$$
\begin{aligned}
u(t)-u(s)= & u^{i_{t}}(t)-u^{i_{s}}(s) \\
= & \left(u^{i_{t}}(t)-u^{i_{t}}\left(T_{i_{t}-1}\right)\right)+\left(u^{i_{t}}\left(T_{i_{t}-1}\right)-u^{i_{t}-1}\left(T_{i_{t}-2}\right)\right)+\cdots \\
& +\left(u^{i_{s}}\left(T_{i_{s}}\right)-u^{i_{s}}(s)\right)
\end{aligned}
$$

and therefore

$$
\begin{aligned}
\|u\|_{\beta, \sim} & \leq\left\|u^{0}\right\|_{\beta, \sim, I_{0}}+\sum_{i=1}^{i^{*}} \sup _{t \in\left[T_{i-1}, T_{i}\right]}\left|u^{i}(t)\right|+T^{\beta} \sum_{i=1}^{i^{*}}\left\|u^{i}\right\|_{\beta, I_{i}} \\
& \leq\left\|u^{0}\right\|_{\beta, \sim, I_{0}}+\max \left(1, T^{\beta}\right) \sum_{i=1}^{i^{*}}\left\|u^{i}\right\|_{\beta, I_{i}}<\infty .
\end{aligned}
$$

Lemma 18. The iterated concatenation $U=(u, v)$ of $U^{i}=\left(u^{i}, v^{i}\right)$ for $i=0,1, \ldots, i^{*}$ (in the sense of Definition 14) verifies $U \in \hat{W}_{0, T}$.

Proof. Since $U^{i} \in \hat{X}_{T_{i-1}, T_{i}, u^{i-1}\left(T_{i-1}\right)}\left(\right.$ and $\left.U^{0} \in \hat{W}_{0, T_{0}}\right)$ there exists $U_{n}^{i}=\left(u_{n}^{i},\left(u_{n}^{i} \otimes \omega_{n}\right)\right)$ such that $u_{n}^{i} \in C_{\gamma}([0, T] ; V)$, with $u_{n}^{i}\left(T_{i-1}\right) \in V_{\gamma},\left(\omega_{n},\left(\omega_{n} \otimes_{S} \omega_{n}\right)\right)$ fulfilling (H3), such that

$$
\lim _{n \rightarrow \infty}\left(u_{n}^{i},\left(u_{n}^{i} \otimes \omega_{n}\right)\right)=\left(u^{i}, v^{i}\right) \quad \text { in } C_{\beta}\left(I_{i} ; V\right) \times C_{\beta+\beta^{\prime}}\left(\bar{\Delta}_{I_{i}} ; V \otimes V\right) ;
$$

Copyright (C) by SIAM. Unauthorized reproduction of this article is prohibited. 
see (17). When $i=0$, taking $u_{n}^{0}(0) \in V_{\gamma}$,

$$
\lim _{n \rightarrow \infty}\left(u_{n}^{0},\left(u_{n}^{0} \otimes \omega_{n}\right)\right)=\left(u^{0}, v^{0}\right) \quad \text { in } C_{\beta, \sim}\left(I_{0} ; V\right) \times C_{\beta+\beta^{\prime}, \sim}\left(\Delta_{I_{0}} ; V \otimes V\right) .
$$

Moreover, thanks to Theorem 16 we can choose for these approximating sequences the classical solutions

$$
\left(u_{n}^{i},\left(u_{n}^{i} \otimes \omega_{n}\right)\right)=U_{n}^{i}=\mathcal{T}\left(U_{n}^{i}, \omega_{n},\left(\omega_{n} \otimes_{S} \omega_{n}\right), u_{n}^{i}\left(T_{i-1}\right)\right) .
$$

Furthermore, according to Definition 14, we can consider the concatenation $U_{n}=\left(u_{n}, v_{n}\right)$. Then $u_{n}$ can be seen as the classical solution to (2) driven by the piecewise linear continuous path $\omega_{n}$ on $[0, T]$. It is easy to see that $u_{n} \in C_{\beta, \sim}([0, T] ; V)$, and by the smoothness of $\omega_{n}$

$$
v_{n}(s, t)=\left(u_{n} \otimes \omega_{n}\right)(s, t)=\int_{s}^{t}\left(u_{n}(r)-u_{n}(s)\right) \otimes_{V} d \omega_{n}
$$

such that $U_{n}$ satisfies the operator equation

$$
U_{n}=\mathcal{T}\left(U_{n}, \omega_{n},\left(\omega_{n} \otimes_{S} \omega_{n}\right), u_{n}(0)\right)
$$

on $[0, T]$, where $\left(u_{n}(0)\right)_{n \in \mathbb{N}}$ tends to $u_{0}$ in $V$. Let us show that

$$
\lim _{n \rightarrow \infty}\left\|U_{n}-U\right\|_{W}=0
$$

which in particular implies that $U \in \hat{W}_{0, T}$. To this end, let us focus on the norm of the difference of area components. In fact, similarly to the proof of Lemma 17, we have

$$
\begin{aligned}
\left\|v-v_{n}\right\|_{\beta+\beta^{\prime}, \sim} \leq & \left\|v^{0}-v_{n}^{0}\right\|_{\beta+\beta^{\prime}, \sim, \Delta_{I_{0}}}+\left\|u^{0}-u_{n}^{0}\right\|_{\beta, \sim, I_{0}}\|\omega\|_{\beta^{\prime},[0, T]} \\
& +\left\|u_{n}^{0}\right\|_{\beta, \sim, I_{0}}\left\|\omega_{n}-\omega\right\|_{\beta^{\prime},[0, T]} \\
& +T^{\beta} \sum_{i=1}^{i^{*}}\left\|v^{i}-v_{n}^{i}\right\|_{\beta+\beta^{\prime}, \bar{\Delta}_{I_{i}}}+T^{\beta} \sum_{i=1}^{i^{*}-1}\left\|u^{i}-u_{n}^{i}\right\|_{\beta, I_{i}}\|\omega\|_{\beta^{\prime},[0, T]} \\
& +T^{\beta} \sum_{i=1}^{i^{*}-1}\left\|u_{n}^{i}\right\|_{\beta, I_{i}}\left\|\omega_{n}-\omega\right\|_{\beta^{\prime},[0, T]},
\end{aligned}
$$

so it suffices to take into account the convergence properties of the beginning of this lemma to conclude that $v_{n} \rightarrow v$ in $C_{\beta+\beta^{\prime}, \sim}\left(\Delta_{0, T}, V \otimes V\right)$. In a similar way we obtain

$$
\lim _{n \rightarrow \infty}\left\|u_{n}-u\right\|_{\beta, \sim}=0 .
$$

Finally, we can state the global existence result when starting with an initial condition in $V$.

Theorem 19. Suppose hypothesis $(\mathrm{H})$ holds and that $\gamma+\beta^{\prime}>1, \gamma \leq 1$, and $u_{0} \in V$. Then there exists a unique global solution $U=(u, v) \in \hat{W}_{0, T}$ to $\mathcal{T}(U)=\bar{U}$. Therefore, the path component $u$ is a global solution to (2).

Proof. By Theorem 11, we obtain the existence of a (local) mild solution $U^{0}=\left(u^{0}, v^{0}\right)$ in $\hat{W}_{0, T_{0}}$ for some $T_{0}>0$. A key feature at this point is that the path component evaluated in 
the final instant belongs to a more regular space, since the solution satisfies that $u^{0}\left(T_{0}\right) \in V_{\gamma}$; see Theorem 11.

Then taking $u_{0}^{1}=u^{0}\left(T_{0}\right) \in V_{\gamma}$ as initial data, we can construct $U^{i}=\left(u^{i}, v^{i}\right), i=1, \ldots, i^{*}$, according to Theorem 16, that later on will be all concatenated turning into $U=(u, v)$. This $U$ belongs to $\hat{W}_{0, T}$ thanks to Lemma 18. In order to finish the proof we check that

$$
U=\mathcal{T}\left(U, \omega,\left(\omega \otimes_{S} \omega\right), u_{0}\right) .
$$

This relationship follows since by Lemma 10(i)

$$
\begin{aligned}
& \left\|\mathcal{T}\left(U, \omega,\left(\omega \otimes_{S} \omega\right), u_{0}\right)-\mathcal{T}\left(U_{n}, \omega_{n},\left(\omega_{n} \otimes_{S} \omega_{n}\right), u_{n}(0)\right)\right\|_{W} \\
& \leq\left\|\mathcal{T}\left(U, \omega,\left(\omega \otimes_{S} \omega\right), u_{0}\right)-\mathcal{T}\left(U_{n}, \omega,\left(\omega \otimes_{S} \omega\right), u_{0}\right)\right\|_{W} \\
& \quad+\left\|\mathcal{T}\left(U_{n}, \omega,\left(\omega \otimes_{S} \omega\right), u_{0}\right)-\mathcal{T}\left(U_{n}, \omega_{n},\left(\omega_{n} \otimes_{S} \omega_{n}\right), u_{0}\right)\right\|_{W} \\
& \quad+\left\|\mathcal{T}\left(U_{n}, \omega_{n},\left(\omega_{n} \otimes_{S} \omega_{n}\right), u_{0}\right)-\mathcal{T}\left(U_{n}, \omega_{n},\left(\omega_{n} \otimes_{S} \omega_{n}\right), u_{n}(0)\right)\right\|_{W} \\
& \leq c T^{\beta^{\prime}}\left(1+\|U\|_{W}^{2}+\left\|U_{n}\right\|_{W}^{2}\right)\left\|U-U_{n}\right\|_{W} \\
& \quad+\left\|\mathcal{T}\left(U_{n}, \omega,\left(\omega \otimes_{S} \omega\right), u_{0}\right)-\mathcal{T}\left(U_{n}, \omega_{n},\left(\omega_{n} \otimes_{S} \omega_{n}\right), u_{0}\right)\right\|_{W} \\
& \quad+\tilde{c}\left|u_{0}-u_{n}(0)\right| .
\end{aligned}
$$

From (30) and Lemma 10(ii) we can conclude that the right-hand-side of the last inequality tends to zero due to the fact that $\left(\left\|U_{n}\right\|_{W}\right)_{n \in \mathbb{N}}$ is bounded. Hence (31) follows from (29) and (30). This mild path-area solution is unique in $\hat{W}_{0, T}$; see [11].

6. Random dynamical systems. In this section we prove that (2) generates a random dynamical system. The fact that a stochastic differential equation generates a random dynamical system allows us to use a huge machinery to investigate qualitative properties of the solution of such a differential equation. We refer to the monograph by Arnold [1].

We consider a metric dynamical system $(\Omega, \mathcal{F}, \mathbb{P}, \theta)$, where $(\Omega, \mathcal{F}, \mathbb{P})$ is a probability space and $\theta$ is a $\mathcal{B}(\mathbb{R}) \otimes \mathcal{F}, \mathcal{F}$-measurable flow on $\Omega$, that is,

$$
\theta_{0}=\mathrm{id}_{\Omega}, \quad \theta_{t} \theta_{s}=\theta_{t+s}, t, s \in \mathbb{R} .
$$

Definition 20. Let $V$ be some topological space. A random dynamical system over the metric dynamical system $(\Omega, \mathcal{F}, \mathbb{P}, \theta)$ is a $\mathcal{B}\left(\mathbb{R}^{+}\right) \otimes \mathcal{F} \otimes \mathcal{B}(V), \mathcal{B}(V)$-measurable mapping such that the cocycle property holds:

$$
\varphi\left(t, \omega, u_{0}\right)=\varphi\left(t-\tau, \theta_{\tau} \omega, \varphi\left(\tau, \omega, u_{0}\right)\right), \quad \varphi\left(0, \omega, u_{0}\right)=u_{0},
$$

for all $t \geq \tau \in \mathbb{R}^{+}, u_{0} \in V$, and $\omega \in \Omega$.

For our application we will choose for $V$ the Hilbert space introduced in section 2. A metric dynamical system is a model for a noise and in this paper we are interested in a fractional noise in $V$ of trace class. Given a probability space and $H \in(0,1)$, a continuous centered Gaussian-process $\beta^{H}(t), t \in \mathbb{R}$, with the covariance function

$$
\mathbb{E} \beta^{H}(t) \beta^{H}(s)=\frac{1}{2}\left(|t|^{2 H}+|s|^{2 H}-|t-s|^{2 H}\right), \quad t, s \in \mathbb{R},
$$

Copyright $\odot$ by SIAM. Unauthorized reproduction of this article is prohibited. 
is called a two-sided one-dimensional fBm, and $H$ is the Hurst parameter. Let $Q$ be a positive symmetric operator of trace class on $V$, i.e., $\operatorname{tr}_{V} Q<\infty$, with positive discrete spectrum $\left(q_{i}\right)_{i \in \mathbb{N}}$ and eigenelements $\left(f_{i}\right)_{i \in \mathbb{N}}$. For simplicity, we assume that $f_{i}=e_{i}$. Then a continuous $V$-valued $f B m B^{H}$ with covariance operator $Q$ and Hurst parameter $H$ is defined by

$$
B^{H}(t)=\sum_{i=1}^{\infty} \sqrt{q_{i}} e_{i} \beta_{i}^{H}(t), \quad t \in \mathbb{R},
$$

where $\left(\beta_{i}^{H}(t)\right)_{i \in \mathbb{N}}$ is a sequence of stochastically independent one-dimensional $\mathrm{fBm}$. It is known that the canonical probability space $\left(C_{0}(\mathbb{R}, V), \mathcal{B}\left(C_{0}(\mathbb{R}, V)\right), \mathbb{P}_{H}, \theta\right)$ is a metric dynamical system where $\mathbb{P}_{H}$ is the fractional Gauss-measure with Hurst parameter $H \in(0,1)$ and determined by $Q$, and $C_{0}(\mathbb{R}, V)$ is the space of continuous paths in $V$ with value zero at zero equipped with the compact open topology. For $\theta$ we take the Wiener-shift given by

$$
\theta_{t} \omega(\cdot)=\omega(\cdot+t)-\omega(t), \quad t \in \mathbb{R}, \omega \in \Omega .
$$

We restrict this metric dynamical system to the set $\Omega$ of $\beta^{\prime \prime}$-Hölder-continuous paths on $[-m, m]$ for any $m \in \mathbb{N}$ and for $1 / 3<\beta^{\prime}<\beta^{\prime \prime}<H \leq 1 / 2$ and equip this set with the $\sigma$-algebra $\mathcal{F}$ given by the $\sigma$-algebra $\mathcal{B}\left(C_{0}(\mathbb{R}, V)\right)$ with respect to $\Omega$, and we restrict $\mathbb{P}_{H}$ to this new domain. The set $\Omega \in \mathcal{B}\left(C_{0}(\mathbb{R}, V)\right)$, it has full measure, and it is $\theta$-invariant. Moreover, the quadruple $\left(\Omega, \mathcal{F}, \mathbb{P}_{H}, \theta\right)$ is a metric dynamical system. For details we refer to [4], [2], or [12].

Note that above, in the definition of $\Omega$, we have picked a new parameter $\beta^{\prime \prime}$ such that $1 / 3<\beta^{\prime}<\beta^{\prime \prime}<H \leq 1 / 2$. By assumption (H3) we can obtain a set $\Omega$ satisfying this regularity condition. The explanation for which we introduce this new parameter $\beta^{\prime \prime}$ can be found in [10].

We would like now to apply the pathwise character of the integral given in section 3 to prove the existence of a random dynamical system generated by the mild solution to (2). For this purpose we have to modify slightly (H3), allowing to treat equations driven by an $\mathrm{fBm}$.

Let $C\left(\Delta, L_{2}\left(L_{2}\left(V, V_{\gamma}\right), V \otimes V\right)\right)$ be the separable Fréchet space of continuous functions defined on $\Delta=\left\{(s, t) \in \mathbb{R}^{2}: s \leq t\right\}$ with values in $L_{2}\left(L_{2}\left(V, V_{\gamma}\right), V \otimes V\right)$.

$\left(H 3^{\prime}\right)$ There exists an $\mathcal{F}, \mathcal{B}\left(C\left(\Delta, L_{2}\left(L_{2}\left(V, V_{\gamma}\right), V \otimes V\right)\right)\right)$-measurable random variable $\left(\omega \otimes_{S}\right.$ $\omega)$ having the following properties:

(i) There exists a $\theta$-invariant set of full measure $\tilde{\Omega} \in \mathcal{F}$ such that for any $\tau \in \mathbb{R}$ we have that

$$
\left(\omega \otimes_{S} \omega\right)(\tau+\cdot, \tau+\cdot)=\left(\theta_{\tau} \omega \otimes_{S} \theta_{\tau} \omega\right)(\cdot, \cdot) .
$$

(ii) For any $m \in \mathbb{N}$ and $\omega$ contained in the invariant set of full measure $\Omega^{\prime} \subset \tilde{\Omega}$ we have

$\lim _{n \rightarrow \infty}\left(\omega_{n},\left(\omega_{n} \otimes_{S} \omega_{n}\right)\right)=\left(\omega,\left(\omega \otimes_{S} \omega\right)\right)$ in $C_{\beta^{\prime}}([-m, m] ; V) \times C_{2 \beta^{\prime}}\left(\bar{\Delta}_{-m, m} ; L_{2}\left(L_{2}(V, \hat{V}), V \otimes V\right)\right)$,

where $\left(\omega_{n}\right)_{n \in \mathbb{N}}$ is a sequence of piecewise linear approximations of $\omega$ with respect to an equidistant dyadic $2^{-n}$ partition and values in $V$.

From now on, outside the invariant set $\Omega^{\prime}$ we define the elements $\left(\omega,\left(\omega \otimes_{S} \omega\right)\right) \equiv 0$.

Note that the first part of this hypothesis holds when considering $\omega$ to be a path of an fBm with $H \in(1 / 3,1 / 2]$; see [10, Theorem 10.4]. In fact, we can prove that there exists 
a strongly stationary version of $\left(\omega \otimes_{S} \omega\right)$ which is indistinguishable from the version stated in $[10$, Theorem 10.1] with respect to $\tilde{\Omega}$, which we denote by the same symbol, and such that

$$
\left(\theta_{\tau} \omega \otimes_{S} \theta_{\tau} \omega\right)(\cdot, \cdot)=\left(\omega \otimes_{S} \omega\right)(\cdot+\tau, \cdot+\tau) .
$$

In particular, the $\theta$-invariance of $\tilde{\Omega}$ follows easily by Lederer [14] and Kümmel [18].

By Deya, Nevenkirch, and Tindel [7] we have that for any $m \in \mathbb{N}$ all conditions from (H3) hold if we replace the interval $[0, T]$ by $[-m, m]$ with respect to a $\theta$-invariant set.

Since $\left(\mathrm{H} 3^{\prime}\right)$ implies (H3), under the assumptions (H1)-(H2) and $\left(\mathrm{H} 3^{\prime}\right)$, thanks to Theorem 19, there exists a global solution $u$ to (2) when the driving process is an fBm with $H \in(1 / 3,1 / 2]$.

On the other hand, the relationship of $\left(\mathrm{H} 3^{\prime}\right)(\mathrm{i})$ also holds if we replace $\omega$ by $\omega_{n}$. Then on a full set $\Omega^{\prime} \subset \tilde{\Omega}$ for $\tau \in \mathbb{R}$ we have that

$$
\left(\omega_{n} \otimes_{S} \omega_{n}\right)(\cdot+\tau, \cdot+\tau)-\left(\omega \otimes_{S} \omega\right)(\cdot+\tau, \cdot+\tau)=\left(\theta_{\tau} \omega_{n} \otimes_{S} \theta_{\tau} \omega_{n}\right)-\left(\theta_{\tau} \omega \otimes_{S} \theta_{\tau} \omega\right) .
$$

Hence the left-hand-side converges iff the right-hand-side converges. By (H3')(ii) the lefthand-side converges to zero on $C_{2 \beta^{\prime}}\left(\bar{\Delta}_{-m, m} ; L_{2}\left(L_{2}(V, \hat{V}), V \otimes V\right)\right)$ for any $m \in \mathbb{N}$ and hence

$$
\left(\omega_{n} \otimes_{S} \omega_{n}\right)(\cdot, \cdot)-\left(\omega \otimes_{S} \omega\right)(\cdot, \cdot)
$$

tends to zero on any $\bar{\Delta}_{-m, m}$. Thus we have the convergence of the right-hand-side on the $\theta$-invariant set $\Omega^{\prime}$ of full measure.

Moreover, from Theorem 19 we know that for any $T$ there exists a unique solution $U$ with respect to the domain $[0, T], \Delta_{0, T}$. We may extend this solution to $\mathbb{R}^{+}, \Delta_{0, \infty}$. In particular, take $T=T_{n}=n$; then the pair $(u(t), v(s, t))$ is given by the solution $U$ with respect to $[0, n], \Delta_{0, n}$, where $t<n$. By the uniqueness result, the restriction of a solution from $[0, T], \Delta_{0, T}$ to $\left[0, T^{\prime}\right], \Delta_{0, T^{\prime}}, T^{\prime}<T$, is a solution on the later domain. Hence this extension makes sense.

Theorem 21. Assume $(\mathrm{H} 1)-(\mathrm{H} 2)$ and $\left(\mathrm{H} 3^{\prime}\right)$. Let $U=(u, v)$ be the solution from Theorem 19 extended to $\mathbb{R}^{+}, \Delta_{0, \infty}$ for $\omega \in \Omega^{\prime}$ with initial condition $u_{0} \in V$. Then u generates a random dynamical system on $\mathbb{R}^{+} \times \Omega^{\prime} \times V$.

Proof. Since the restriction of $U$ to $[0, T], \Delta_{0, T}$ is in $\hat{W}_{0, T}$, it follows that the integral $\mathcal{T}_{1}(U)$ is additive. Hence $U=(u, v)$ restricted to $[\tau, T], \Delta_{\tau, T}$ solves

$$
U=\mathcal{T}^{\tau}\left(U, \omega,\left(\omega \otimes_{S} \omega\right), \mathcal{T}_{1}\left(U, \omega,\left(\omega \otimes_{S} \omega\right), u_{0}\right)(\tau)\right)
$$

uniquely for $\tau>0 \omega \in \Omega^{\prime}$, and $u_{0} \in V$. Setting

$$
\varphi\left(t, \omega, u_{0}\right):=\mathcal{T}_{1}\left(U, \omega,\left(\omega \otimes_{S} \omega\right), u_{0}\right)(t)=u(t)
$$

we obtain the cocycle property if

$$
\mathcal{T}_{1}\left(U, \omega,\left(\omega \otimes_{S} \omega\right), u_{0}\right)(t)=\mathcal{T}_{1}\left(U_{\tau}, \theta_{\tau} \omega,\left(\theta_{\tau} \omega \otimes_{S} \theta_{\tau} \omega\right), u(\tau)\right)(t-\tau),
$$

where $U_{\tau}(s, t)=\left(u_{\tau}, v_{\tau}\right)(s, t)=(u(\tau+t), v(\tau+s, \tau+t))$. Note that because $u, v$, and $\omega$ are related by the Chen property (8), the same happens for $u_{\tau}, v_{\tau}$, and $\theta_{\tau} \omega$.

Copyright (c) by SIAM. Unauthorized reproduction of this article is prohibited. 
For the classical evolution equation (3) we get

$$
\begin{aligned}
& \mathcal{T}_{1}\left(\left(u_{n}(\cdot),\left(u_{n} \otimes \omega_{n}\right)(\cdot, \cdot)\right), \omega_{n},\left(\omega_{n} \otimes_{S} \omega_{n}\right), u_{n}(0)\right)(t) \\
& =\mathcal{T}_{1}^{\tau}\left(\left(u_{n}(\cdot),\left(u_{n} \otimes \omega_{n}\right)(\cdot, \cdot)\right), \omega_{n},\left(\omega_{n} \otimes_{S} \omega_{n}\right), u_{n}(\tau)\right)(t-\tau) \\
& =\mathcal{T}_{1}\left(\left(u_{n}(\cdot+\tau),\left(u_{n} \otimes \omega_{n}\right)(\cdot+\tau, \cdot+\tau)\right), \theta_{\tau} \omega_{n},\left(\theta_{\tau} \omega_{n} \otimes_{S} \theta_{\tau} \omega_{n}\right), u_{n}(\tau)\right)(t-\tau) \\
& =\mathcal{T}_{1}\left(\left(u_{n}(\cdot+\tau),\left(u_{n}(\cdot+\tau) \otimes \theta_{\tau} \omega_{n}\right)(\cdot, \cdot)\right), \theta_{\tau} \omega_{n},\left(\theta_{\tau} \omega_{n} \otimes_{S} \theta_{\tau} \omega_{n}\right), u_{n}(\tau)\right)(t-\tau)
\end{aligned}
$$

for every $\omega \in \Omega^{\prime}$ because

$$
\left(\omega_{n}\right)^{\prime}(\cdot+\tau)=\left(\theta_{\tau} \omega_{n}\right)^{\prime}(\cdot)
$$

Note that in the last formulas we could omit $\left(\theta_{\tau} \omega_{n} \otimes_{S} \theta_{\tau} \omega_{n}\right)$ because the classical solutions are independent of this term. Moreover, above we have used that the $\mathcal{T}_{2}$-component given by $\left(u_{n} \otimes \omega_{n}\right)($ see $(10))$ has the property

$$
\left(u_{n} \otimes \omega_{n}\right)(\cdot+\tau, \cdot+\tau)=\left(u_{n}(\cdot+\tau) \otimes \theta_{\tau} \omega_{n}\right)(\cdot, \cdot) .
$$

Therefore, since $U \in \hat{W}_{0, T}$, the convergence conclusion of Theorem 19, which holds for every $\omega \in \Omega^{\prime}$ independently of $u_{0}$, applied to the left-hand and right-hand-sides of (34) yields (33), and therefore the cocycle property is established for $\varphi$.

Now we deal with the measurability of $\varphi$. Consider (32) replacing $\left(\omega,\left(\omega \otimes_{S} \omega\right)\right)$ by $\left(\omega_{n},\left(\omega_{n} \otimes_{S} \omega_{n}\right)\right)$. The mapping $\omega \mapsto \omega_{n}$ is $\mathcal{F}, \mathcal{F}$-measurable for every $n \in \mathbb{N}$ and hence the mapping $\omega \mapsto\left(\omega_{n} \otimes_{S} \omega_{n}\right)$ is $\mathcal{F}, \mathcal{B}\left(C\left(\Delta, L_{2}\left(L_{2}\left(V, V_{\gamma}\right), V \otimes V\right)\right)\right.$-measurable. Let $U_{n}=\left(u_{n}, v_{n}\right)$ be the solution for parameters $\left(\omega_{n},\left(\omega_{n} \otimes_{S} \omega_{n}\right)\right)$. Then $u_{n}$ is the classical solution to (2) (and $\left.v_{n}=\left(u_{n} \otimes \omega_{n}\right)\right)$. Hence for the first component of $U_{n}$ we have that

$$
[0, T] \times \Omega^{\prime} \times V \ni\left(t, \omega, u_{0}\right) \mapsto u_{n}(t) \in V
$$

is measurable with respect to $\mathcal{B}([0, T]) \otimes \mathcal{F} \otimes \mathcal{B}(V), \mathcal{B}(V)$. However, from (30)

$$
\hat{W}_{0, T}-\lim _{n \rightarrow \infty} U_{n}=U
$$

and hence for the first components of $U$ and $U_{n}$ we have for every $t \in[0, T]$

$$
\lim _{n \rightarrow \infty} u_{n}(t)=u(t)
$$

which gives the measurability of $u(t)$ with respect to $\mathcal{F} \otimes \mathcal{B}(V)$. Since $u(t)$ is continuous in $t$ we obtain by $(32)$ the $\mathcal{B}([0, T]) \otimes \mathcal{F} \otimes \mathcal{B}(V), \mathcal{B}(V)$-measurability, that is, measurability restricted to $[0, T]$, see Castaing and Valadier [3, Chapter III]. Considering an increasing sequence $\left(T_{n}\right)_{\in \mathbb{N}}, T_{n}>0$, with limit $\infty$ and setting

$$
u_{n}(t)= \begin{cases}u(t) & : t \leq T_{n}, \\ u\left(T_{n}\right) & : \quad t \geq T_{n},\end{cases}
$$

then $u_{n}$ is $\mathcal{B}\left(\mathbb{R}^{+}\right) \otimes \mathcal{F} \otimes \mathcal{B}(V), \mathcal{B}(V)$-measurable and for any $\left(t, \omega, u_{0}\right)$ the mapping $u_{n}$ converges to the first component $u$ of the extended solution $U$. Hence $\varphi$ is $\mathcal{B}\left(\mathbb{R}^{+}\right) \otimes \mathcal{F} \otimes \mathcal{B}(V), \mathcal{B}(V)$ measurable. 
Remark 22. There is another way to prove that the path-area solution generates a random dynamical system which is based on Kager and Scheutzow [16]. In particular (34) gives us the random flow property for the $u$-component of the path-area solution. The conditions of $[16$, Theorem 4] to generate a cocycle from a (semi)flow are fulfilled. The measurability condition (iii') in that article follows (for instance) because $\mathcal{T}_{1}^{\tau}$ is a pointwise limit of the classical solution having these measurability properties; ( $\left.\mathrm{v}^{\prime}\right)$ is due to the continuity of $t \mapsto \mathcal{T}_{1}^{\tau}(t)$ and (iv') follows from the additivity of the pathwise integral and the continuous dependence of $\mathcal{T}$ on the initial condition.

7. Example. Let us assume that $A$ is generated by the Laplacian on $\mathcal{O}=(0,1)$ with homogeneous Dirichlet boundary condition. $A$ with domain $D(-A)=H^{2}(\mathcal{O}) \cap H_{0}^{1}(\mathcal{O})$ generates a semigroup in $L_{2}(\mathcal{O})$. Let $\rho=1 / 4+\epsilon, \epsilon>0$, small. Then $V:=D\left((-A)^{\rho}\right)$ consists of the Slobodetski spaces $H^{2 \rho}(\mathcal{O})$ satisfying the homogeneous boundary conditions; see Da Prato and Zabczyk [5, p. 401]. In particular, the continuous embedding $V \subset C(\overline{\mathcal{O}})$ holds. In what follows we consider the restriction of the semigroup $S$ to the space $V$. Note that the inequalities (4) and (5) continue being true and that $\left(\lambda_{i}^{-\rho} e_{i}\right)_{i \in \mathbb{N}}$ is an orthonormal basis of $V$, where $\left(\lambda_{i}\right)_{i \in \mathbb{N}}$ is the spectrum of $A$ and $\left(e_{i}\right)_{i \in \mathbb{N}}$ are the associated eigenelements of $A$ with respect to $L_{2}(\mathcal{O})$, which are uniformly bounded in $L_{\infty}(\mathcal{O})$. The asymptotical behavior of the spectrum is given by $\lambda_{i} \sim i^{2}$.

Lemma 23. For $\mu \in(1,5 / 4)$

$$
D\left((-A)^{\mu}\right)=H^{2 \mu}(\mathcal{O}) \cap H_{0}^{1}(\mathcal{O}) .
$$

Proof. On $H^{2 \mu}(\mathcal{O}) \cap H_{0}^{1}(\mathcal{O})$ we know that $A=\Delta_{H D B C}$, which is an isomorphism with range $H^{2 \mu-2}(\mathcal{O})$; see Egorov and Shubin [8, p. 124 ]. In addition, $(-A)^{\mu-1}$ has the domain $H^{2 \mu-2}(\mathcal{O})$ if $\mu \in(1,5 / 4)$; see [5, p. 401].

Now for $1 / 3<\beta^{\prime}<1 / 2$ we take

$$
\eta>1-\beta^{\prime}, \quad \gamma=\eta+\rho<1
$$

That choice of $\eta$ and $\gamma$ ensures that we can define $\left(\omega \otimes_{S} \omega\right)$ as in [10, Theorem 10.1] (see also Lemma 10.2 of that paper). In addition, we can ensure the assumptions of the global existence Theorem 18 since the above choice implies $\gamma+\beta^{\prime}>1$.

Let $g$ be four times continuously differentiable on $\mathcal{O}$, where the derivatives can be extended continuously on $\overline{\mathcal{O}}$, and assume that $g$ is zero on $\{0,1\} \times \mathbb{R}$, such that all the corresponding derivatives ( $g$ itself included) are bounded. Define

$$
G(u)(v)[x]=\int_{\mathcal{O}} g(x, u(y)) v(y) d y \quad \text { for } u, v \in V .
$$

Following Kantorowitsch and Akilow [17, section XVII.3], it is not hard to prove that $G$ is three times continuously differentiable where the derivatives are given by

$$
D G(u)\left(v, h_{1}\right)[x]=\int_{\mathcal{O}} D_{2} g(x, u) v(y) h_{1}(y) d y,
$$

Copyright $\odot$ by SIAM. Unauthorized reproduction of this article is prohibited. 


$$
\begin{aligned}
D^{2} G(u)\left(v, h_{1}, h_{2}\right)[x] & =\int_{\mathcal{O}} D_{2}^{2} g(x, u) v(y) h_{1}(y) h_{2}(y) d y, \\
D^{3} G(u)\left(v, h_{1}, h_{2}, h_{3}\right)[x] & =\int_{\mathcal{O}} D_{2}^{3} g(x, u) v(y) h_{1}(y) h_{2}(y) h_{3}(y) d y
\end{aligned}
$$

for $v, h_{1}, h_{2}, h_{3} \in V$. By the previous choice of the parameters, $\rho+\gamma=2 \rho+\eta \in(1,5 / 4)$, hence by Lemma $23 V_{\gamma}:=D\left((-A)^{\rho+\gamma}\right)=D\left((-A)^{2 \rho+\eta}\right)=H^{2(2 \rho+\eta)}(\mathcal{O}) \cap H_{0}^{1}(\mathcal{O})$, and then $G(u)(v), D G(u)\left(v, h_{1}\right), D^{2} G(u)\left(v, h_{1}, h_{2}\right), D^{3} G(u)\left(v, h_{1}, h_{2}, h_{3}\right) \in H^{3}(\mathcal{O}) \cap H_{0}^{1}(\mathcal{O}) \subset V_{\gamma}$. For instance, by the continuous embedding theorem we have that

$$
\begin{aligned}
& \int_{\mathcal{O}} \mid \int_{\mathcal{O}} D_{2}^{2} D_{1}^{k} g\left(x, u(y)+h_{3}(y)\right) v(y) h_{1}(y) h_{2}(y)-D_{2}^{2} D_{1}^{k} g(x, u(y)) v(y) h_{1}(y) h_{2}(y) \\
& \quad-\left.D_{2} D_{2}^{2} D_{1}^{k} g(x, u(y)) v(y) h_{1}(y) h_{2}(y) h_{3}(y) d y\right|^{2} d x \leq c\left(\int_{\mathcal{O}}\left|v(y) h_{1}(y) h_{2}(y)\right|\left|h_{3}(y)\right|^{2} d y\right)^{2} \\
& \quad \leq c^{\prime}|v|_{C}^{2}\left|h_{1}\right|_{C}^{2}\left|h_{2}\right|_{C}^{2}\left|h_{3}\right|_{C}^{4} \leq c^{\prime \prime}|v|^{2}\left|h_{1}\right|^{2}\left|h_{2}\right|^{2}\left|h_{3}\right|^{4} \quad \text { for } k=1,2,3,
\end{aligned}
$$

where $c^{\prime}$ is a uniform bound for $\left|D_{2}^{4} D_{1}^{k} g(x, u)\right|^{2}|\mathcal{O}|^{2}$. This gives the differentiability of $D^{2} G(u)$ in $H^{3}(\mathcal{O}) \cap H_{0}^{1}(\mathcal{O})$ and since $G(u)(v)[x], \ldots, D^{3} G(u)\left(v, h_{1}, h_{2}, h_{3}\right)[x]$ are zero for $x \in\{0,1\}$ we have differentiability in $V_{\gamma}$ too.

The Hilbert-Schmidt property of $D G(u)$ follows by

$$
\sup _{k=1,2,3} \sum_{i, j} \int_{\mathcal{O}}\left(\int_{\mathcal{O}} \mid D_{2} D_{1}^{k} g(x, u(y)) \lambda_{i}^{-\rho} e_{i}(y) \lambda_{j}^{-\rho} e_{j}(y) d y\right)^{2} d x<c\left(\sum_{i} \lambda_{i}^{-2 \rho}\right)^{2}<\infty
$$

due to the boundedness of $D_{2} D_{1}^{k} g$ and the uniform boundedness of $\left(e_{i}\right)_{i \in \mathbb{N}}$ in $L_{\infty}(\mathcal{O})$. In the same manner we obtain that the other derivatives are Hilbert-Schmidt operators.

These estimates allow us to apply Theorem 19 to SEEs that have the above kernel integral diffusion operator. For a different example of diffusion we refer the reader to [11].

Acknowledgment. The authors would like to acknowledge the referee for his/her careful reading of the text and his/her comments and suggestions.

\section{REFERENCES}

[1] L. Arnold, Random Dynamical Systems, Springer Monogr. Math., Springer-Verlag, Berlin, 1998.

[2] T. Caraballo, M. J. Garrido-Atienza, B. Schmalfuss, and J. Valero, Asymptotic behavior of a stochastic semilinear dissipative functional equation without uniqueness of solutions, Discrete Contin. Dyn. Syst. Ser. B, 14 (2010), pp. 439-455.

[3] C. Castaing and M. Valadier, Convex Analysis and Measurable Multifunctions, Lecture Notes in Math. 580, Springer-Verlag, Berlin, 1977.

[4] Y. Chen, H. Gao, M. J. Garrido-Atienza, and B. Schmalfuss, Pathwise solutions of SPDEs and random dynamical systems, Discrete Contin. Dyn. Syst. Ser. A, 34 (2014), pp. 79-98.

[5] G. Da Prato And J. ZabczyK, Stochastic Equations in Infinite Dimensions, Cambridge University Press, Cambridge, UK, 1992.

[6] A. Deya, M. Gubinelli, And S. Tindel, Non-linear rough heat equations, Probab. Theory Related Fields, 153 (2012), pp. 97-147.

Copyright (C) by SIAM. Unauthorized reproduction of this article is prohibited. 
[7] A. Deya, A. Neuenkirch, And S. Tindel, A Milstein-type scheme without Lévy area terms for SDES driven by fractional Brownian motion, Ann. Inst. H. Poincaré Probab. Statist., 48 (2012), pp. 518-550.

[8] Y. V. Egorov And M. A. Shubin, Foundations of the Classical Theory of Partial Differential Equations, Encyclopaedia Math. Sci. 30. Springer, New York, 1998.

[9] M. J. Garrido-Atienza, K. Lu, and B. Schmalfuss, Compensated fractional derivatives and stochastic evolution equations, C. R. Math., 350 (2012), pp. 1037-1042.

[10] M. J. Garrido-Atienza, K. Lu, and B. Schmalfuss, Lévy Areas of Ornstein-Uhlenbeck Processes in Hilbert Spaces, in Continuous and Distributed Systems II, Theory and Applications, Stud. Systems Decision Control 30, Springer, New York, 2015, pp. 167-188.

[11] M. J. Garrido-Atienza, K. Lu, And B. Schmalfuss, Local pathwise solutions to stochastic evolution equations driven by fractional Brownian motions with Hurst parameters $H \in(1 / 3,1 / 2]$, Discrete Contin. Dyn. Syst. Ser. B, 20 (2015), pp. 2553-2581.

[12] M. J. Garrido-Atienza and B. Schmalfuss, Ergodicity of the infinite dimensional fractional Brownian motion, J. Dynam. Differential Equations, 23 (2011), pp. 671-681.

[13] Y. Hu And D. Nualart, Rough path analysis via fractional calculus, Trans. Amer. Math. Soc., 361 (2009), pp. 2689-2718.

[14] C. LEDERER, Konjugation stochastischer und zufälliger stationärer Differentialgleichungen und eine Version des lokalen Satzes von Hartman-Grobman für stochastische Differentialgleichungen, Ph.D. thesis, HU Berlin, 2001.

[15] R. V. Kadison And J. R. Ringrose, Fundamentals of the Theory of Operator Algebras: Elementary Theory, Grad. Stud. Math., AMS, Providence, RI, 1997.

[16] G. Kager And M. Scheutzow, Generation of one-sided random dynamical systems by stochastic differential equations, Electron. J. Probab., 2 (1997), pp. 1-17.

[17] L. W. Kantorowitsch AND G. P. Akilow, Funktionalanalysis in normierten Räumen, Verlag Harri Deutsch, 1978.

[18] K. KüMMEL, Dynamics of Marcus type SDEs, in preparation.

[19] H. Kunita, Stochastic Flows and Stochastic Differential Equations, Cambridge University Press, Cambridge, UK, 1990.

[20] T. Lyons And Z. Qian, Flow of diffeomorphisms induced by a geometric multiplicative functional, Probab. Theory Related Fields, 112 (1991), pp. 91-119.

[21] B. Maslowski and D. Nualart, Evolution equations driven by a fractional Brownian motion, J. Funct. Anal., 202 (2003), pp. 277-305.

[22] A. PAZy, Semigroups of Linear Operators and Applications to Partial Differential Equations, Springer Appl. Math. Ser., Springer-Verlag, Berlin, 1983.

[23] M. Scheutzow, On the perfection of crude cocycles, Random Comput. Dynam., 4 (1996), pp. 235-255.

Copyright (C) by SIAM. Unauthorized reproduction of this article is prohibited. 